\title{
Impact of the second-order self-forces on the dephasing of the gravitational waves from quasicircular extreme mass-ratio inspirals
}

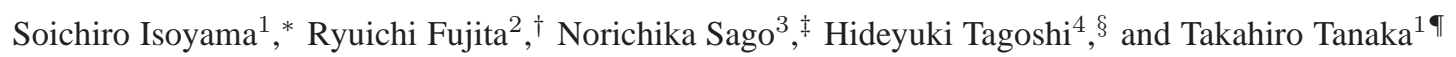 \\ ${ }^{1}$ Yukawa Institute for Theoretical Physics, Kyoto university, Kyoto, 606-8502, Japan \\ ${ }^{2}$ Departament de Física, Universitat de les Illes Balears, Palma de Mallorca, E-07122 Spain \\ ${ }^{3}$ Faculty of Arts and Science, Kyushu University, Fukuoka 819-0395, Japan \\ ${ }^{4}$ Department of Earth and Space Science, Graduate School of Science, Osaka Univeristy, Osaka, 560-0043, Japan
}

(Dated: September 26, 2018)

\begin{abstract}
The accurate calculation of the long-term phase evolution of gravitational wave (GW) forms from extreme (intermediate) mass-ratio inspirals [E(I)MRIs] is an inevitable step to extract information from this system. In order to achive this goal, it is believed that we need to understand the gravitational self-forces. However, it has not been quntatively demonstrated that the second-order self-forces are necessary for this purpose. In this paper we revisit the problem to estimate the order of magnitude of the dephasing caused by the secondorder self-forces on a small body in a quasicircular orbit around a Kerr black hole, based on the knowledge of the post-Newtonian (PN) approximation and invoking the energy balance argument. In particular, we focus on the averaged dissipative part of the self-force, since it gives the leading-order contribution among their various components. To avoid the possibility of the energy flux of GWs becoming negative, we propose a new simple resummation called exponential resummation, which assures the positivity of the energy flux. In order to estimate the magnitude of the yet-unknown second-order self-forces, here we point out the scaling property in the absolute value of the PN coefficients of the energy flux. Using these new tools, we evaluate the expected magnitude of dephasing. Our analysis indicates that the dephasing due to the second-order self-forces for quasicircular E(I)MRIs may be well captured by the $3 \mathrm{PN}$ energy flux, once we obtain all the spin-dependent terms, except for the case with an extremely large spin of the central Kerr black hole.
\end{abstract}

PACS numbers: 04.30Db, 04.25Nx, 04.30Tv, 95.85.Sz

\section{INTRODUCTION}

Extreme mass-ratio inspirals (EMRIs) and intermediate mass-ratio inspirals (IMRIs), in which a stellar mass or a compact object with several tens solar masses inspirals into a more massive central black hole, have attracted much interest not only as a promising source of the gravitational waves (GWs) for future spaceborne $\mathrm{GW}$ detectors, but also as a unique clean probe of the spacetime region of strong gravity. To achieve the test of general relativity using GWs from E(I)MRIs, we need to predict sufficiently accurate waveforms. This requirement motivates us to model the E(I)MRIs as the motion of a small body in a given background spacetime with gravitational backreaction. This backreaction is treated as gravitational self-forces [1-6], and its higher-order extension with respect to the mass-ratio has attracted much interest in recent years [7-11]. See the following review articles, e.g., Refs. [12, 13] and references therein for more details.

In fact, following the scaling argument (See, e.g., Refs.[14, 15]), the phase of GWs from a particle whose orbital frequency sweeps a few orders of magnitude before the plunge can be expanded as

$$
\Phi=\frac{M_{\mathrm{BH}}}{\mu}\left[\Phi^{(0)}+\frac{\mu}{M_{\mathrm{BH}}} \Phi^{(1)}+O\left(\frac{\mu^{2}}{M_{\mathrm{BH}}^{2}}\right)\right],
$$

where $\Phi^{(0)}$ and $\Phi^{(1)}$ are $O(1)$ quantities independent of $\mu$, which is the mass of a small particle, and $M_{\mathrm{BH}}$ and $a$ are the mass and the spin parameter of the Kerr black hole, respectively. ${ }^{1}$ On the one hand $\Phi^{(0)}$ in Eq. (1), we only need the self-forces up to the first-order time-averaged dissipative part. In addition, it has long been known that $\Phi^{(0)}$ can be computed with the well-established balance argument, which relates the first-order time-averaged dissipative part of the self-forces to the energy and angular momentum fluxes associated with global Killing vectors on the background Kerr black hole[20]. Though the Carter

\footnotetext{
*Electronic address: isoyama at yukawa.kyoto-u.ac.jp

${ }^{\dagger}$ Electronic address: ryuichi.fujita'at' uib.es

${ }^{\ddagger}$ Electronic address: sago 'at artsci.kyushu-u.ac.jp

§Electronic address: tagoshi at vega.ess.sci.osaka-u.ac.jp

IElectronic address: tanaka'at yukawa.kyoto-u.ac.jp

${ }^{1}$ Throughout this paper, we assume that the resonance is absent during its orbital evolution. See Refs. 15- 19] for further discussion about resonant orbits.
} 
constant is not associated with any Killing vector (and thus the simple balance argument is not applicable), the way to computing long time averaged evolution of the Carter constant has already been well established[21-24]. On the other hand, $\Phi^{(1)}$, which is referred to as the dephasing relative to $\Phi^{(0)}$, depends on two different components of the self-forces: the first-order conservative part, and the averaged second-order dissipative part. ${ }^{2}$ The first subleading term, $\Phi^{(1)}$, can still be important, since potentially it may give a correction significantly greater than unity to the phase [25-29].

In the effort to obtain an accurate waveform, there have been many works on self-forces. As for the first-order conservative part, thanks to recent massive development, we are now in part ready for practical computation with numerical implementation [30-34]. Particularly, in the case of the Schwarzschild background, the corrections to the orbital frequencies [35] and $\Phi^{(1)}$ [36] have already been studied extensively. Even in the case of the Kerr background preliminary results of the self-forces in quasicircular orbits have been reported[37].

By contrast, the averaged second-order dissipative part of the self-forces has so far been studied only at the formal level in the context of black hole perturbation [7-11]. It will require much more effort to establish the method for computing the secondorder dissipative part, especially in the case of the Kerr background. Under such circumstances, a typical strategy for evaluating $\Phi^{(1)}$ is to make use of the standard post-Newtonian (PN) approximation, in which we assume slow motion of a satellite and its weak gravitational field. Based on the PN approximation, Huerta and Gair[38] evaluated the size of the dephasing caused by the first-order conservative self-forces and the averaged second-order self-forces, picking up representative EMRIs in quasicircular orbits on a Kerr black hole. The same dephasing was also discussed by Yunes et al. [39], using the effective one-body formalism again for representative E(I)MRIs.

A naive expectation is that the PN approximation will not suitable for modelling the waveforms of E(I)MRIs, especially for a Kerr black hole with large spin. Typical E(I)MRIs in circular orbits spend the last few years of inspiral in the vicinity of the inner most stable circular orbit (ISCO). Since the ISCO radius reaches the event horizon as the spin of the Kerr black hole is increased to the extremal limit, the motion of the body becomes highly relativistic, exceeding the validity range of the standard PN approximation[?]. However, things are not so trivial. The time spent near the ISCO becomes longer as we increase the mass ratio $M_{\mathrm{BH}} / \mu$, but in that case the mass of the large central black hole also becomes larger. Then, the total cycles of GWs become smaller for a given observation time. As a result, the correction due to higher-order self-forces might be suppressed below the observational threshold, despite the loss of accuracy of the PN approximation. Therefore, it is not so obvious whether there are really E(I)MRIs that require the notion of the second-order self-forces.

The previous analyses mentioned above [38, 39] are focusing on the corrections coming from the self-forces at the currently available PN order and are limited to representative E(I)MRIs. To get an insight into whether or not the second-order self-forces based on the black hole perturbation are really necessary to calculate the waveforms of quasicircular E(I)MRIs, therefore, it would be useful to give an estimate of dephasing coming from the averaged second-order dissipative self-forces, focusing on the yet-unknown higher PN terms and surveying the whole parameter region of E(I)MRIs.

What we discuss in this paper is the adiabatic evolution of E(I)MRIs in quasicircular orbits on a Kerr spacetime. Here the adiabatic evolution means an approximation in which the evolution of the orbital frequency is determined by the energy balance argument; i.e., the rate of change of the total energy of the binary is equated to the energy flux emitted to infinity.

To evaluate the order of magnitude of the yet-unknown higher PN corrections, we need to rely on some extrapolation. For this purpose, we first introduce a simple new resummation of the energy flux, which we call the "exponential resummation." When the spin of a Kerr black hole is large enough, the PN energy flux in the Taylor form can be negative outside the ISCO radius for some PN orders [41]. If this happens, the estimated total phase before the plunge diverges and the extrapolation to the higher PN order will not make sense. Our simple "exponential resummation" is the one that ensures the positivity of the energy flux.

As a PN input for the corrections at the next leading order in the mass ratio, ${ }^{3}$ the best one available so far is the 3.5PN energy flux of GWs[43, 44] with linear spin-dependent terms up to the $3 \mathrm{PN}$ order, which has recently been derived by Blanchet $e t$ al. [45]. To estimate the possible magnitude of the yet-unknown higher-order PN terms, we focus on a scaling property among the PN coefficients in the energy flux. Using the 8 PN energy flux in the test particle limit [46? ], we will show that the absolute values of the coefficients scale roughly as required from the convergence of the PN series up to the light-ring radius. (This point was also discussed by Nakano et al. independently[47].) Since this scaling behavior is related to the PN convergence, despite the lack of the higher PN terms in the energy flux, we conjecture that the same scaling property will hold for the higher-order

\footnotetext{
${ }^{2}$ Here the "dissipative" part refers to the self-forces that cause the time variation of the constants of motion, such as the energy, the angular momentum around the axis of symmetry, and the Carter constant. The "conservative" part is the part that gives the correction to the relation between the orbital frequencies and the constants of motion [15, 21]. The meaning of "time-averaged" is averaging over a sufficiently long period compared to the time scale for the evolution of the phase difference between the oscillations in the radial and the zenith angle directions.

3 Throughout this paper, we use the notion "-mass ratio" to refer to the symmetric mass ratio $\nu:=\mu M_{\mathrm{BH}} /\left(M_{\mathrm{BH}}+\mu\right)^{2}$, instead of the usual mass ratio $\mu / M_{\mathrm{BH}}$. We should note that the terms higher-order in the mass ratio arise even at the level of the quadrupole formula if we use another mass ratio. In this sense, definitely the use of the symmetric mass ratio is advantageous. This fact has indeed been taken into account in the previous analyses such as that of Le Tiec et al. [42], where the periastron advance due to the conservative portion of the first-order self-forces is compared to the result deduced from the numerical relativity.
} 
terms in the mass-ratio. Under this assumption, we estimate the order of magnitude of the unknown portion of the energy flux coming from higher PN terms at the next leading order in the mass ratio via "extrapolation." Gathering the tools stated above, we will investigate the impact on the dephasing from the averaged dissipative second-order self-forces for various E(I)MRIs systematically. This is the main goal of this paper.

The remainder of this manuscript is organized as follows: In Sec. II we briefly review how the accumulated phase of GWs from adiabatic inspiral is calculated based on the balance argument. In Sec. IIII to cure the negative energy flux of GWs that appears in the truncated PN Taylor series expansion, we propose the exponential resummation that ensures the positivity of the energy flux. Section IV is dedicated to the study of the scaling property in the coefficients of the energy flux in the test particle limit that becomes manifest owing to the brand-new 8 PN energy flux [? ]. In Sec.V] using the exponential resummation and the scaling property, we will estimate the dephasing coming from the yet-unknown part of the second-order self-forces for various E(I)MRIs. We find that the unkown non-linear spin-dependent terms at the lower PN order in the energy flux dominate the unknown dephasing. We summarize our results and conclude in Sec.VI]

In this manuscript, we use geometrical units $G=c=1$, and the sign convention of the metric is $(-,+,+,+)$. The coordinates $(t, r, \theta, \phi)$ denote the Boyer-Lindquist coordinates of the Kerr black hole[48]. We frequently use the dimensionless spin defined by $q:=a / M_{\mathrm{BH}}$, and the symmetric mass ratio defined by $\nu:=\mu M_{\mathrm{BH}} /\left(M_{\mathrm{BH}}+\mu\right)^{2}$.

\section{THE ACCUMULATED PHASE OF THE GRAVITATIONAL WAVE FROM AN INSPIRALING BINARY}

We consider a binary composed of a small satellite body with the rest mass $\mu$ in a quasicircular orbit around a Kerr black hole with the mass $M_{\mathrm{BH}}$ and the spin parameter $a$. We neglect the effect of the spin of the satellite, which may be negligiblly small for the detection stage of E(I)MRIs, though it plays no negligible role in their parameter estimation [49, 50]. For a binary in a quasicircular orbit, the accumulated phase of GWs is calculated as

$$
\Phi:=-2 \int_{x_{\mathrm{ISCO}}}^{x_{0}} d x \frac{x^{3 / 2}}{M} \frac{E^{\prime}(x)}{\dot{E}(x)},
$$

where $E$ is the binding energy of the binary, $\dot{E}:=d E / d t$ is the energy loss rate, and the prime denotes the differentiation with respect to $x$, which is a dimensionless orbital frequency defined by $x:=(M \Omega)^{2 / 3}$, with the orbital frequency $\Omega$ and the total mass $M:=M_{\mathrm{BH}}+\mu$. Here $E$ is supposed to depend only on $x$, neglecting the effect of the variation of mass due to the energy absorbed by the black hole. Throughout the paper we totally neglect the effect of this time-dependent mass variation. We choose the lower bound of the integral in Eq. (2), $x_{\mathrm{ISCO}}$, to be the value of $x$ at the inner most stable circular orbit (ISCO), and the upper bound, $x_{0}$, to be the value determined by the condition coming from the finite observation time. Throughout this paper, we adopt $t_{\mathrm{obs}}=1 \mathrm{yr}$ as the observation time.

Our primary interest in this paper is the order of magnitude of the dephasing coming from the corrections at the next leading order in the mass ratio. To evaluate it, we calculate the difference between $\Phi$ with and without the higher-order corrections. In checking the detectability of this difference by observation, the initial and the final frequencies should be kept unchanged when we evaluate it. Here, we fix both $x_{0}$ and $x_{\text {ISCO }}$ to the values determined by the test particle limit. Namely, [51]

$$
\begin{aligned}
x_{\mathrm{ISCO}} & :=\left(R_{\mathrm{ISCO}}^{3 / 2}+q\right)^{-1 / 6}, \quad R_{\mathrm{ISCO}}:=3+Z_{2} \mp \sqrt{\left(3-Z_{1}\right)\left(3+Z_{1}+2 Z_{2}\right)}, \\
Z_{1} & :=1+\left(1-q^{2}\right)^{1 / 3}\left[(1+q)^{1 / 3}+(1-q)^{1 / 3}\right], \quad Z_{2}:=\sqrt{3 q^{2}+Z_{1}^{2}},
\end{aligned}
$$

where the upper (lower) sign in the second equation is chosen for the co- (counter-)rotating case with $q>(<) 0$, and $R:=$ $r / M_{\mathrm{BH}}$ represents the dimensionless radius. $x_{0}$ is determined by

$$
1(\mathrm{yr})=-\int_{x_{\mathrm{ISCO}}}^{x_{0}} d x \frac{E^{\prime[0]}(x)}{\dot{E}^{[0]}(x)},
$$

where the superscript ${ }^{[0]}$ means the leading-order contribution in the mass ratio, i.e., the test particle limit, and

$$
E^{[0]}=\mu \frac{R^{3 / 2}-2 R^{1 / 2}+q}{R^{3 / 4}\left(R^{3 / 2}-3 R^{1 / 2}+2 q\right)^{1 / 2}}, \quad \Omega:=\frac{1}{M\left(R^{3 / 2}+q\right)}, \quad R=\left(\frac{1-q x^{3 / 2}}{x^{3 / 2}}\right)^{2 / 3} .
$$

As for the energy, we adopt here the binding energy of the binary instead of the energy directly related to the four-momentum of the particle. It would be more natural to consider the latter in the context of the self-force calculation based on the black hole perturbation, but it is not gauge invariant. Hence, the former is more suitable for comparison with the PN calculation. These two 
energies are different at the next leading order in the mass ratio due to the presence of the gravitational field energy, but there must be one-to-one correspondence between them, once the gauge is completely fixed.

The energy balance argument tells us that the averaged loss of the total energy should be equal to the averaged total energy flux to the future null infinity because of energy conservation (see, e.g., Refs. [20, 52]). Hence, as long as we define the binding energy appropriately,

$$
-\frac{d E}{d t}=\mathcal{L}
$$

holds, in the sense averaged over a sufficiently long time and neglecting the horizon absorption flux, where $\mathcal{L}$ is the energy flux emitted to the null infinity. Hence, we evaluate the phase by

$$
\Phi[\mathcal{L}]:=2 \int_{x_{\mathrm{ISCO}}}^{x_{0}} d x \frac{x^{3 / 2}}{M} \frac{E^{\prime[0]}(x)}{\mathcal{L}(x)} .
$$

In the above equation, as we are interested in the dissipative corrections, we fix $E^{\prime}$ to the expression in the test particle limit. We expand $\mathcal{L}$ as $\mathcal{L}=\mathcal{L}^{[0]}+\nu \mathcal{L}^{[1]}+O\left(\nu^{2}\right)$. To compute $\mathcal{L}^{[0]}$, we can use the Teukolsky formalism[53] and invoke the numerical code developed by Fujita and Tagoshi[54, 55].

The finite mass corrections at the next leading order, $\mathcal{L}^{[1]}$, are in part provided by the standard PN calculations. In the PN formalism, the energy flux of GWs emitted to infinity from a quasicircular binary is obtained up to the 3.5PN order for the spin-independent terms [43, 44] and up to the $3 \mathrm{PN}$ order for the terms linear in spin when the spin vectors are parallel to the orbital axis [45]. In the present notation, truncated at $O(\nu)$, it is given by

$$
\begin{aligned}
\mathcal{L}_{n \mathrm{PN}}^{\mathrm{T}}(x, q)= & \frac{32}{5} x^{5} \nu^{2}\left[1+x\left(-\frac{1247}{336}-\frac{35}{12} \nu\right)+x^{3 / 2}\left(4 \pi-\frac{11}{4} q+\frac{17}{4} q \nu\right)+x^{2}\left(-\frac{44711}{9072}+\frac{9271}{504} \nu\right)\right. \\
& +x^{5 / 2}\left(-\frac{8191}{672} \pi-\frac{59}{16} q+\nu\left\{-\frac{583}{24} \pi+\frac{3749}{144} q\right\}\right) \\
& +x^{3}\left(\frac{6643739519}{69854400}+\frac{16}{3} \pi^{2}-\frac{1712}{105} \gamma_{\mathrm{E}}-\frac{856}{105} \log (16 x)-\frac{65}{6} \pi q+\left\{-\frac{134543}{7776}+\frac{41}{48} \pi^{2}+\frac{33}{2} \pi q\right\} \nu\right) \\
& \left.+x^{7 / 2}\left(-\frac{16285}{504}+\frac{214745}{1728} \nu\right) \pi+O\left(x^{4}, \nu^{2}, q^{2}, q x^{7 / 2}\right)\right]
\end{aligned}
$$

where $\gamma_{\mathrm{E}}=0.57721 \ldots$ denotes Euler's constant. We will refer to the above expression for the energy flux truncated at the $n$th PN order ${ }^{4}$ as "the $n \mathrm{PN}$ Taylor flux." We denote it by $\mathcal{L}_{n \mathrm{PN}}^{\mathrm{T}}$, and expand it like $\mathcal{L}_{n \mathrm{PN}}^{\mathrm{T}}:=\mathcal{L}_{n \mathrm{PN}}^{\mathrm{T}[0]}+\nu \mathcal{L}_{n \mathrm{PN}}^{\mathrm{T}[1]}+\cdots$ as before. We also frequently use the "normalized $n \mathrm{PN}$ flux" defined by

$$
L_{n \mathrm{PN}}^{\mathrm{T}}(x, q):=\left(\frac{32}{5} \nu^{2} x^{5}\right)^{-1} \mathcal{L}_{n \mathrm{PN}}^{\mathrm{T}}(x, q) .
$$

From now on, we denote the PN flux solely composed of the known part as "the known $n$ PN Taylor flux" and distinguish it from the full PN flux with the notation, as with $\tilde{\mathcal{L}}_{n \mathrm{PN}}^{\mathrm{T}}$. We also introduce notation to denote the residual terms of higher-order in PN expansion and in spin:

$$
\mathcal{L}_{>n \mathrm{PN}}^{\mathrm{T}[i]}:=\mathcal{L}_{\text {full }}^{[i]}-\mathcal{L}_{n \mathrm{PN}}^{\mathrm{T}[i]}
$$

Then, $\tilde{\mathcal{L}}_{>3.5 \mathrm{PN}}^{\mathrm{T}[1]}$ represents the yet-unknown energy flux to be determined from the computation of the second-order dissipative self-forces. Further we define $\tilde{\mathcal{L}}_{n \mathrm{PN}}^{\mathrm{T}[0]}$ and $\tilde{\mathcal{L}}_{>n \mathrm{PN}}^{\mathrm{T}[0]}$ by the terms in $\mathcal{L}^{[0]}$ that correspond to $\tilde{\mathcal{L}}_{n \mathrm{PN}}^{\mathrm{T}[1]}$ and $\tilde{\mathcal{L}}_{>n \mathrm{PN}}^{\mathrm{T}[1]}$, respectively. To be precise, we define $\tilde{\mathcal{L}}_{n \mathrm{PN}}^{\mathrm{T}[0]}$ by the sum of the spin-independent terms up to the 3.5PN order and the terms linear in the black hole spin up to the $3 \mathrm{PN}$ order. $\tilde{\mathcal{L}}_{>n \mathrm{PN}}^{\mathrm{T}[0]}$ signifies the remaining terms at leading order in the mass ratio, which also includes the nonlinear spin-dependent terms in all PN orders. ${ }^{5}$.

\footnotetext{
4 We refer to terms of $O\left(x^{n}\right)$ relative to the leading order as those of $n \mathrm{PN}$ order.

${ }^{5}$ Here we do not distinguish the logarithmic term in the 3 PN Taylor flux in the test particle limit from the other 3 PN terms. We have confirmed that the results in the present paper do not change much even if we exclude this term from $\tilde{\mathcal{L}}_{n \mathrm{PN}}^{\mathrm{T}[0]}$
} 


\section{A MANIFESTLY POSITIVE DEFINITE ENERGY FLUX: EXPONENTIAL RESUMMATION}

In this section we propose a new simple resummation scheme, which can be easily applied if we just know the $n$ PN Taylor flux.

\section{A. Limitation of the Taylor flux revisited}

It is easy to imagine that the PN Taylor flux is not so accurate when the orbital radius becomes small. Indeed, Zhang et al.[? ] pointed out that the $n \mathrm{PN}$ Taylor flux in the test particle limit rapidly loses accuracy around the ISCO radius. However, it is a different issue whether the Taylor flux is accurate enough for our present purpose because what we are interested in here is whether or not the dephasing in GW waveforms is measurbale.

As we want to evaluate the correction to $\Phi$ in Eq. (7) caused by the yet-unknown part of the flux $\tilde{\mathcal{L}}_{>n \text { PN }}^{\mathrm{T}[1]}$, we need some extrapolation method. For this purpose, it will later become necessary to evaluate something like the phase for the flux truncated at the $n \mathrm{PN}$ order, $\Phi\left[\tilde{\mathcal{L}}_{n \mathrm{PN}}^{\mathrm{T}[0]}\right]$. At this point, we find that the $n \mathrm{PN}$ Taylor flux is problematic. As mentioned earlier, the energy flux truncated at some PN orders becomes negative outside $R_{\mathrm{ISCO}}$ if the spin of black hole $q$ is sufficiently large, firstly pointed out by Tagoshi et al. [41].

To see how serious the problem is, we revisit the Taylor flux in the test particle limit. The spin-independent terms in $\mathcal{L}_{n \mathrm{PN}}^{\mathrm{T}[0]}$ have been analytically calculated up to the $22 \mathrm{PN}$ order and the spin-dependent terms up to the 8 PN order by Fujita 46 ? ]. The expression of the normalized $8 \mathrm{PN}$ energy flux including complete spin-dependent terms is schematically expanded as 6

$$
L_{8 \mathrm{PN}}^{\mathrm{T}[0]}(x, q)=\sum_{n=0}^{8} \sum_{p=0}^{p_{\max }} \hat{L}^{(n, p)}(q) x^{n}(\log (x))^{p},
$$

where $p_{\max }$ is the maximum integer that does not exceed $n / 3$. In Fig. 11 we depict $L_{n \mathrm{PN}}^{\mathrm{T}[0]}(x, q)$, the normalized $n \mathrm{PN}$ Taylor flux in the test particle limit truncated at various PN orders for $q=0.9$. The curves terminate at the ISCO frequency. We also plot the exact numerical energy flux in the test particle limit as a reference. The exact numerical energy flux is manifestly positive definite for $x<x_{\text {ISCO }}(0.9)$, while all the $n$ PN Taylor fluxes in Fig. 1 cross zero at some $x=x_{0}(q)<x_{\text {ISCO }}(0.9)$. In addition to the already-known 2.5 PN and 4 PN cases ${ }^{7}$ [41], we also find that the $5 \mathrm{PN}, 5.5 \mathrm{PN}, 6.5 \mathrm{PN}$, and 8 PN fluxes become negative before $x$ reaches $x_{\mathrm{ISCO}}(q)$. Even for a moderate value such as $q=0.7$, we still observe the flux to cross zero, say at order $8 \mathrm{PN}$. Once this happens, the integrand of (7) diverges, and then the phase evaluated by using the truncated flux $\mathcal{L}_{n \mathrm{PN}}^{\mathrm{T}[0]}$ does not make sense. For a reliable extrapolation, it is necessary to use a resummed expression for the energy flux that gives at least a finite estimate of the phase for the truncation at any PN order.

\section{B. The exponential resummation and an improved hybrid energy flux}

To overcome the drawback of the $n \mathrm{PN}$ Taylor flux, a naive requirement will be to guarantee that the energy flux is always positive by resummation. Among various known resummation techniques [56-59], to the best of our knowledge, only the factorized resummation ensures the positivity of the energy flux. This resummation was proposed by Damour et al. [60] for the equal-mass nonspinning binaries in a circular orbit. Fujita and Iyer[61] and Pan et al. [62] applied it to a test particle in a circular orbit around a Schwarzschild and a Kerr black hole, respectively. Here, we propose another even simpler resummation, which is defined by

$$
\mathcal{L}_{n \mathrm{PN}}^{\exp }(x, q):=\frac{32}{5} \nu^{2} x^{5} \exp \left[L_{n \mathrm{PN}}^{\exp }(x, q)\right]
$$

with

$$
L_{n \mathrm{PN}}^{\exp }(x, q):=\left.\log \left[L_{n^{\prime} \mathrm{PN}}^{\mathrm{T}}(x, q)\right]\right|_{\text {truncated at } n \mathrm{PN} \text { order }}
$$

\footnotetext{
6 The explicit expression for $\mathcal{L}_{8 \mathrm{PN}}^{\mathrm{T}[0]}$ will be made available elsewhere in an appropriate form[? ].

${ }^{7}$ While Tagoshi et al. [41] reported that the 3 PN Taylor flux with $q=0.9$ became negative for small radii, we find that the 3 PN Taylor flux is always positive definite for all radii, irrespective of the spin parameter of the Kerr black hole.
} 


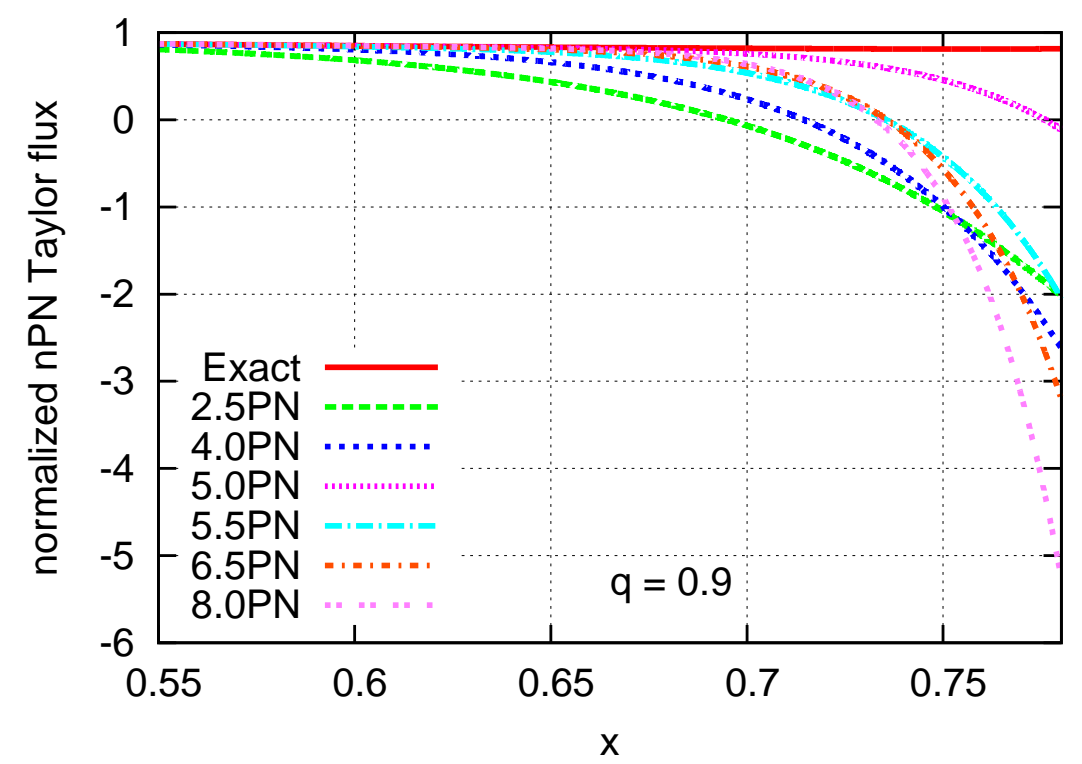

FIG. 1: The normalized $n \mathrm{PN}$ Taylor flux in the test particle limit, $L_{n \mathrm{PN}}^{\mathrm{T}[0]}(x, q)$, for $q=0.9$ up to $x_{\mathrm{ISCO}}(0.9):=0.78014 \ldots$ The horizontal axis is the dimensionless frequency $x$ of a body. The label "Exact" means the exact numerical energy flux, calculated by Fujita and Tagoshi[54].

where $n^{\prime} \geq n$ is understood. We refer to this resummation and the energy flux [Eq. [12] ] as the "exponential resummation" and the " $n \mathrm{PN}$ exponential resummed flux," respectively. This flux is manifestly positive and can incorporate any higherorder corrections if the counterparts in the $n \mathrm{PN}$ Taylor flux are given. Furthermore, it significantly improves the accuracy of approximation [? ]. For definiteness, we explicitly write the definitions of $\mathcal{L}_{n \mathrm{PN}}^{\exp [0]}$ and $\mathcal{L}_{n \mathrm{PN}}^{\exp [1]}$ as

$$
\mathcal{L}_{n \mathrm{PN}}^{\exp [0]}(x, q):=\frac{32}{5} \nu^{2} x^{5} \exp \left[L_{n \mathrm{PN}}^{\exp [0]}(x, q)\right], \quad \mathcal{L}_{n \mathrm{PN}}^{\exp [1]}(x, q):=\left(\frac{32}{5} \nu^{2} x^{5} \exp \left[L_{n \mathrm{PN}}^{\exp [0]}(x, q)\right]\right) L_{n \mathrm{PN}}^{\exp [1]}(x, q),
$$

with $L_{n \mathrm{PN}}^{\exp }=L_{n \mathrm{PN}}^{\exp [0]}+\nu L_{n \mathrm{PN}}^{\exp [1]}+\cdots$. We show the explicit form only for $L_{n \mathrm{PN}}^{\exp [1]}$ as

$$
\begin{aligned}
L_{n \mathrm{PN}}^{\exp [1]}(x, q)=- & \frac{35}{12} x+\frac{17}{4} q x^{3 / 2}+\frac{30523}{4032} x^{2}+\left(\frac{136229}{4032} q-\frac{101}{8} \pi\right) x^{5 / 2} \\
& +\left(-\frac{43670915}{12192768}+\frac{41}{48} \pi^{2}-\frac{\pi}{2} q\right) x^{3}+\frac{70075}{6048} \pi x^{7 / 2}+O\left(x^{4}, q x^{7 / 2}, q^{2}\right) .
\end{aligned}
$$

Combining the exact numerical flux obtained in the test particle limit, $\mathcal{L}_{\text {full }}^{[0]}$, and the PN flux, $\mathcal{L}_{\mathrm{PN}}$, one can obtain a better estimate for the energy flux. In the case of the Taylor flux, these two are combined simply by summing $\mathcal{L}_{\text {full }}^{[0]}$ and $\mathcal{L}_{\mathrm{PN}}^{\mathrm{T}[1]}$. In the case of the exponential resummed energy flux [Eq. [12 ] ], we need to do this summation at the level of the exponent, $L^{\exp }$, say, expanding $L_{n \mathrm{PN}}^{\exp }$ in powers of $\nu$, we replace $L_{n \mathrm{PN}}^{\exp [0]}$ with the one corresponding to the numerical flux. Then, we obtain

$$
\mathcal{L}_{n \mathrm{PN}}^{\text {hyb }}(x, q)=\mathcal{L}_{\text {full }}^{[0]}(x, q) \exp \left[\nu L_{n \mathrm{PN}}^{\exp [1]}(x, q)+\cdots\right],
$$

which we call the " $n$ PN hybrid flux."

Analogously, we introduce the "known $n \mathrm{PN}$ exponential resummed flux" and the "known $n$ PN hybrid flux," and distinguish them from their respective counterparts with the notation. Here, the truncation for the known part is made at the level of $L^{\exp [1]}$. Namely, $\tilde{L}_{n \mathrm{PN}}^{\exp [1]}$ is truncated at the same PN order for each order of spin that is included in the known terms $\tilde{\mathcal{L}}_{n \mathrm{PN}}^{\mathrm{T}[1]}$. For the exponential resummed flux, we can also introduce $\tilde{\mathcal{L}}_{n \mathrm{PN}}^{\exp [0]}$, the counterpart of $\tilde{\mathcal{L}}_{n \mathrm{PN}}^{\exp [1]}$ in the test particle limit, as in the case of $\tilde{\mathcal{L}}_{n \mathrm{PN}}^{\mathrm{T}[0]}$. That is, $\tilde{L}_{n \mathrm{PN}}^{\exp [0]}$ is truncated at the same PN order for each order of spin that is included in the known terms $\tilde{\mathcal{L}}_{n \mathrm{PN}}^{\mathrm{T}[1]}$. On the other hand, $\tilde{\mathcal{L}}_{n \mathrm{PN}}^{\text {hyb }[0]}$ does not make sense. In the following discussion, we identify the hybrid flux with the exponential resummed flux for the leading order in the mass ratio. We summarize our notation for various fluxes used in the rest of our paper in Table [ for readability. 


\begin{tabular}{c|c}
\hline \hline & Symbol \\
\hline \hline The flux truncated at $n \mathrm{PN}$ order & $\mathcal{L}_{n \mathrm{PN}}^{[i]}$ \\
The residual part of $\mathcal{L}_{n \mathrm{PN}}^{[i]}$ & $\mathcal{L}_{i n n \mathrm{PN}}^{[i]}$ \\
$\tilde{\mathcal{L}}_{n \mathrm{PN}}^{[i]}$ \\
The known part of the flux truncated at $n \mathrm{PN}$ order & $\tilde{\mathcal{L}}_{>n \mathrm{PN}}^{[i]}$ \\
The residual of $\tilde{\mathcal{L}}_{n \mathrm{PN}}^{[i]}$ & $L_{n \mathrm{PN}}^{\mathrm{T}[i]}$ \\
The normalized $n \mathrm{PN}$ Taylor flux & $L_{n \mathrm{PN}}^{\exp [i]}$ \\
\hline \hline
\end{tabular}

TABLE I: Our notation for various fluxes in this paper. Upper index $[i]$ refers to the order of truncation with respect to the mass ratio $\nu$ : $\mathcal{L}=\mathcal{L}^{[0]}+\nu \mathcal{L}^{[1]}+O\left(\nu^{2}\right)$. We also put label "T", "exp", "'hyb" and "full" to distinguish the flux type. Respectively, they correspond to Taylor, exponential, hybrid and numerical complete flux.

\section{THE SCALING LAW OF THE COEFFICIENTS IN THE ENERGY FLUX}

Now we tackle our main issue: how to evaluate the magnitude of the yet-unknown part of the energy flux, $\tilde{\mathcal{L}}_{>3.5 \mathrm{PN}}^{[1]}$. (When we discuss general issues independent of the form of the energy flux, we simply suppress the labels "T", "exp" and "hyb.") Our strategy here is to establish the scaling property in the coefficients of the PN expansion of the energy flux in the test particle limit for fixed $q$. (This point was also discussed by Nakano et al. independently[47].) The argument of the $n$ PN exponential resummed flux, $L_{n \mathrm{PN}}^{\exp [0]}$, is expanded as

$$
L_{n \mathrm{PN}}^{\exp [0]}(x, q):=\sum_{n}\left(\frac{x}{x_{\operatorname{lr}}(q)}\right)^{n} \sum_{p} C_{n, p}(q)\left(\log \left[\frac{x}{x_{\operatorname{lr}}(q)}\right]\right)^{p},
$$

with

$$
x_{\operatorname{lr}}(q):=\left(\frac{1}{R_{\mathrm{lr}}^{3 / 2}+q}\right)^{2 / 3},
$$

where

$$
R_{\mathrm{lr}}(q):=2\left[1+\cos \left(\frac{2}{3} \arccos q\right)\right]
$$

is the value of $x$ corresponding to the radius of the circular orbit on the light ring[51].

It is known that for circular orbits the source term of the Teukolsky equation has a simple pole when the orbit is on the light-ring radius, i.e., at $x=x_{\mathrm{lr}}(q)[63$, 64]. The light-ring radius is the innermost radius for the presence of an (unstable) circular orbit, and there the particle energy per unit rest mass diverges. As a result, the energy flux also diverges there. Besides the light-ring singularity, another origin of singularity in solving the Teukolsky equation is the presence of quasi-normal mode poles, which are zeros of the Wronskian. However, the absolute value of the first quasi-normal mode frequency, which is the smallest, is almost identical to the frequency of GWs from the source at the light ring[65]. Hence, we expect that $x_{\operatorname{lr}}$ determines, at least approximately, the convergence radius when the energy flux is seen as an analytic function of $x$. If the PN expansion of the energy flux converges as long as $x<x_{\operatorname{lr}}(q)$, then $x=x_{\operatorname{lr}}(q)$ is the first place where the series ceases to converge.

With this expectation in mind, we plot the absolute value contribution from each PN order $\left|C_{n, 0}\right|$ with $x=x_{\operatorname{lr}}(q)$ in Fig. 2 for various values of $q$. Here $q=0.998$ is a bound for possible maximum spin of an astrophysical black hole, called the Thorne limit[66]. As expected, $\left|C_{n, 0}\right|$ is roughly independent of the PN order $n$, as is typical at the boundary of convergence for $|q|<0.9$. Contrary to our expectation, for $|q|>0.9,\left|C_{n, 0}\right|$ continues to increase up to the $8 \mathrm{PN}$, and the series seems to diverge. However, this does not mean that PN series does not converge for $x \approx x_{\operatorname{lr}}(q)$ with $|q| \approx 1$. When we make a similar plot for each partial wave contribution for a higher multipole component, the contribution to the energy flux peaks around a higher PN order, but the series is convergent, although we cannot check extremely high multipole components. On the other hand, the summation over multipole components is known to converge from the numerical computation of the energy flux by Fujita and Tagoshi[54, 55]. These facts may indicate that the convergence depends on the order of summation. The point of Fig. 22 is that $\left|C_{n, 0}\right|$ shows a nice scaling property with respect to the PN order for any value of $q$. Even for $q$ close to the extremal limit, the plot becomes flat if we substitute a slightly smaller value of $x$ instead of $x_{1 \mathrm{r}}$.

Now we are in the position to discuss how to estimate the order of magnitude of the yet-unknown part of the energy flux, $\tilde{\mathcal{L}}_{>3.5 \mathrm{PN}}^{[1]}$. The second-order perturbed Einstein equation would schematically take the form $\square h^{(2)}=(T[z+\delta z]-T[z])+$ $h^{(1)} T[z]+\left(\nabla h^{(1)}\right)^{2}$, where $h^{(1)}$ and $h^{(2)}$ are, respectively, the first- and second-order perturbations induced by a body. Here 


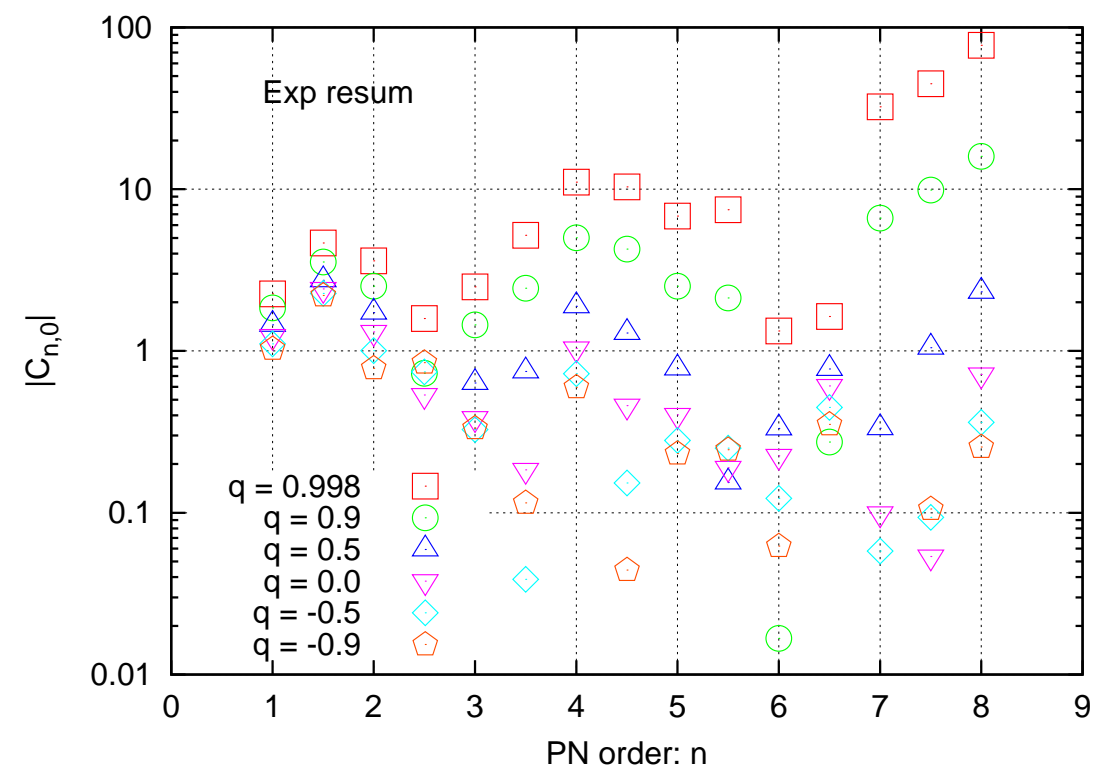

FIG. 2: The absolute value of the contribution to the energy flux from each PN order $\left|C_{n, 0}\right|$ at $x=x_{\operatorname{lr}}(q)$, with the horizontal axis being the PN order $n$. Note that the values of $\left|C_{n, 0}\right|$ are accidentally small with some fixed $q$. We have safely exludeded these values from the plot.

$z$ is the background Kerr geodesic and $\delta z$ is the $O(\nu)$ correction to it [67]. We know that all the first-order perturbations have singularity only at $x \approx x_{\operatorname{lr}}(q)$. The Green's function $\square^{-1}$ is basically the same as in the linear case. Hence, even for the second-order perturbation, the convergence radius in $x$ will be the same as in the linear case, and thus a similar scaling for the PN coefficients will be expected for the next leading order in the mass ratio, too. Then, we can guess the amplitude of the higher PN coefficients from the first few terms in the PN expansion that are known from the standard PN calculation.

One may worry that $\square^{-1}$ has singularities at the quasi-normal mode frequencies. When we consider the second-order perturbation, there may arise high-frequency components which are absent at the level of linear perturbation. However, this might not happen. The point is that the metric perturbation caused by a circular geodesic has a helical Killing vector. ${ }^{8}$ At the level of the second-order source term, this helical symmetry is broken due to the presence of the deviation from the geodesic, $\delta z$. Apart from this contribution, however, the source term keeps the helical symmetry and hence the frequency that appears in a partial wave labeled by $(\ell, m)$ is $m \Omega$ only. Namely, higher frequency modes do not arise. Even if we take into account $\delta z$, the time scale associated with $\delta z$ is as slow as $\Omega^{-1} \times O\left(\nu^{-1}\right)$. Therefore, the presence of $\delta z$ alters the frequency $m \Omega$ only by a small amount of $O(\nu \Omega)$. This essentially does not change the convergence radius in $x$.

Even if we find the order of magnitude of the PN coefficients, the scaling property tells us nothing about the actual sign of each term. Therefore, when $x$ is close to $x_{\operatorname{lr}}(q)$, it becomes difficult to guess the order of the magnitude of the infinite summation. However, since the higher-order PN terms are suppressed by the power of $x / x_{\mathrm{lr}}(q)$, the summation is dominated by a few leading terms when $x / x_{\operatorname{lr}}(q)$ is reasonably small. In that case, we do not have to worry about the infinite summation. Here, we bravely step a little forward by making the following proposal: the ratio between $\tilde{\mathcal{L}}_{n \mathrm{PN}}^{[1]}$ and $\tilde{\mathcal{L}}_{>n \mathrm{PN}}^{[1]}$ will be the same order as the ratio between $\tilde{\mathcal{L}}_{n \mathrm{PN}}^{[0]}-\mathcal{L}_{0 \mathrm{PN}}^{[0]}$ and $\tilde{\mathcal{L}}_{>n \mathrm{PN}}^{[0]}$. Based on this assumption, we extrapolate the known results to estimate the unknown $\tilde{\mathcal{L}}_{>n \mathrm{PN}}^{[1]}$

\section{THE DEPHASING DUE TO THE AVERAGED DISSIPATIVE PORTION OF THE SECOND-ORDER SELF-FORCES}

The main focus of this section is to evaluate the dephasing from the averaged dissipative part of the second-order self-forces in E(I)MRIs, based on the idea of the extrapolation proposed in the preceding section. In this paper, we measure the magnitude

\footnotetext{
8 The helical Killing vector $t^{\alpha}+\Omega \phi^{\alpha}$, that remains to generate a symmetry even for the perturbed spacetime: where $t^{\alpha}$ and $\phi^{\alpha}$ are the asymptotically time translation and rotational Killing vectors of the background Kerr black hole, respectively. See, e.g., Ref.[68] for more detailes.
} 

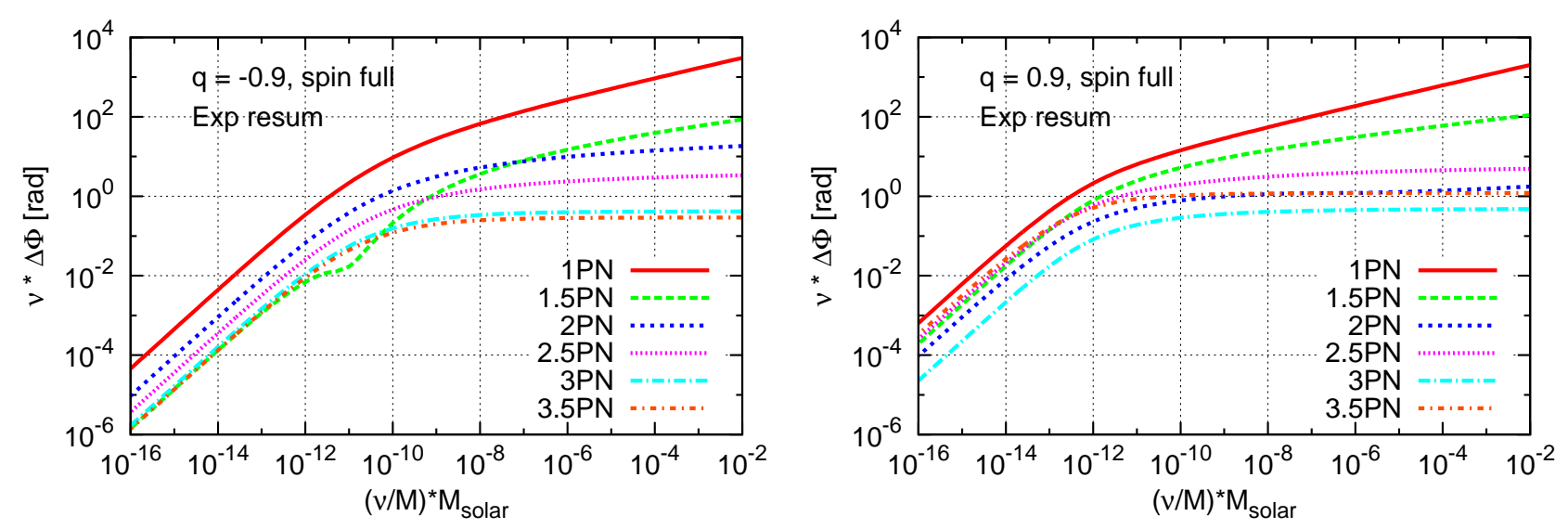

FIG. 3: The dephasing due to the higher PN corrections in the test particle limit $\nu \Delta \Phi\left[\mathcal{L}_{n \mathrm{PN}}^{\exp [0]}, \mathcal{L}_{>n \mathrm{PN}}^{\exp [0]}\right]$ as a function of $\nu / M$ for $q= \pm 0.9$.

of the dephasing due to higher-order corrections to the energy flux $\delta \mathcal{L}$ by

$$
\Delta \Phi[\mathcal{L}, \delta \mathcal{L}]:=-2 \int_{x_{\mathrm{ISCO}}}^{x_{0}} d x \frac{x^{3 / 2} E^{\prime[0]}(x)}{M}\left|\frac{1}{\mathcal{L}(x)+\delta \mathcal{L}(x)}-\frac{1}{\mathcal{L}(x)}\right|
$$

Here the absolute value of the difference of fluxes is taken in the integrand. The factor $(\mathcal{L}+\delta \mathcal{L})^{-1}-\mathcal{L}^{-1}$ can change its signature in the domain of the integral for some parameter region of E(I)MRIs. Therefore, if we do not take the absolute value, there might be an accidental cancellation between positive and negative contributions, and the order of magnitude of the evaluated $\Delta \Phi$ can largely deviate from what we really want to measure. Notice that, even if $\Delta \Phi$ defined without taking the absolute value in the integrand strictly vanishes, the deviation in the GW waveform is still detectable. Therefore, to avoid this possible underestimate of the dephasing, we take the absolute value of the difference.

As a general remark, we would like to mention the mass dependences of $\Phi\left[\mathcal{L}_{\text {ref }}^{[0]}\right]$, the phase for a certain reference flux $\mathcal{L}_{\text {ref }}^{[0]}$ which includes the leading PN terms in the test particle limit, and $\Delta \Phi\left[\mathcal{L}_{\text {ref }}^{[0]}, \delta \mathcal{L}^{[0]}\right]$ and $\Delta \Phi\left[\mathcal{L}_{\text {ref }}^{[0]}, \delta \mathcal{L}^{[1]}\right]$, the dephasings due to $\delta \mathcal{L}^{[0]}$ and $\delta \mathcal{L}^{[1]}$, which are portions of the energy flux at the leading order and the next leading order in the mass ratio, respectively. From the integral in Eq. (4), which defines $x_{0}$, one can factor out $M_{\mathrm{BH}} / \nu$ since

$$
E^{\prime[0]} \propto M_{\mathrm{BH}} \nu, \quad \mathcal{L}_{\mathrm{ref}}^{[0]} \propto \nu^{2}
$$

for a given $x$. Hence, we find that $x_{0}$ is a function of $M_{\mathrm{BH}} / \nu$. Substituting $\delta \mathcal{L}^{[0]} \propto \nu^{2}$ and $\delta \mathcal{L}^{[1]} \propto \nu^{3}$ together with the relations in Eq. (21) into the definitions of $\nu \times \Phi\left[\mathcal{L}_{\text {ref }}^{[0]}\right], \nu \times \Delta \Phi\left[\mathcal{L}_{\text {ref }}^{[0]}, \delta \mathcal{L}{ }^{[0]}\right]$ and $\Delta \Phi\left[\mathcal{L}_{\text {ref }}^{[0]}, \delta \mathcal{L}^{[1]}\right]$, we find that their mass dependences remain only through $x_{0}$. Therefore, one can conclude that $\nu \times \Phi\left[\mathcal{L}_{\text {ref }}^{[0]}\right], \nu \times \Delta \Phi\left[\mathcal{L}_{\text {ref }}^{[0]}, \delta \mathcal{L}^{[0]}\right]$ and $\Delta \Phi\left[\mathcal{L}_{\text {ref }}^{[0]}, \tilde{\mathcal{L}}^{[1]}\right]$ depend on $M_{\mathrm{BH}}$ and $\nu$ only through the combination $M_{\mathrm{BH}} / \nu$ for a large mass ratio $\nu \ll 1$ for a fixed observation period before the plunge.

\section{A. The error caused by the post-Newtonian truncation in the test particle limit}

Before we evaluate the dephasing due to the yet-unknown part of the energy flux at the next leading order in the mass ratio, we will assess the magnitude of the dephasing due to the PN truncation in the test particle limit $\Delta \Phi\left[\mathcal{L}_{n \mathrm{PN}}^{[0]}, \mathcal{L}_{>n \mathrm{PN}}^{[0]}\right]$ in this subsection and that coming from the known PN terms $\Delta \Phi\left[\mathcal{L}_{\text {full }}^{[0]}+\tilde{\mathcal{L}}_{n \mathrm{PN}}^{[1]}, \tilde{\mathcal{L}}_{>n \mathrm{PN}}^{[1]}\right]$ in the succeeding subsection.

Here, we study the quantity $\Delta \Phi\left[\mathcal{L}_{n \mathrm{PN}}^{[0]}, \mathcal{L}_{>n \mathrm{PN}}^{[0]}\right]$ to examine the PN convergence in terms of the phase error for various E(I)MRIs. We show the results only for the exponential resummed flux because the phase for the PN Taylor flux becomes ill defined for several PN orders. (The hybrid flux is identical to the exponential resummed one by definition in the test particle limit.)

In Fig. 3 we plot $\nu \Delta \Phi\left[\mathcal{L}_{n \mathrm{PN}}^{\exp [0]}, \mathcal{L}_{>n \mathrm{PN}}^{\exp [0]}\right]$ as a function of $\nu / M$ for $q= \pm 0.9$. The main message of these plots is that the PN convergence is almost uniform, except for the case of large black hole spin for large $\nu / M$. When $q$ is large, ISCO becomes close to the light-ring radius where PN convergence becomes very slow. Even in that case, for lower PN orders the convergence is rather smooth for large $\nu / M$. This is because up to the $2 \mathrm{PN}$ order, the phase correction is dominated by the lower bound of the $x$-integral in Eq. [20], if the initial separation of the binary at the time $1 \mathrm{yr}$ before the plunge is sufficiently large. The 

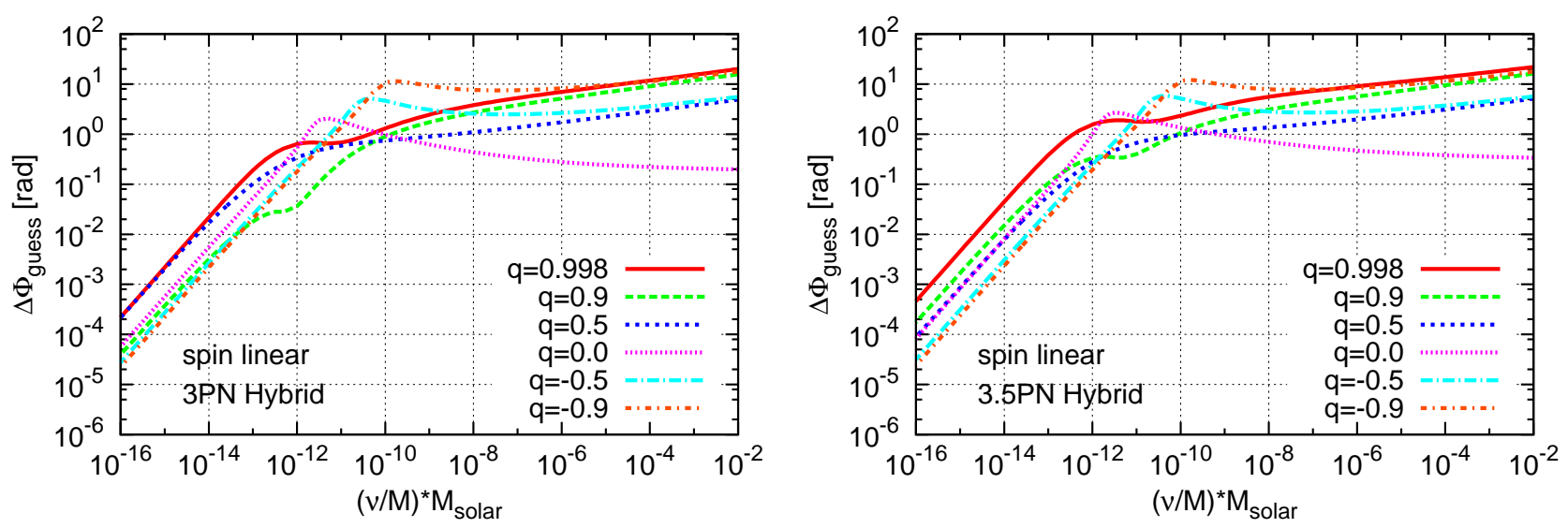

FIG. 4: The expected residual dephasing $\Delta \Phi_{\text {guess }}\left[\mathcal{L}_{\text {full }}^{[0]}+\tilde{\mathcal{L}}_{n \mathrm{PN}}^{[1]}, \tilde{\mathcal{L}}_{>n \mathrm{PN}}^{[1]}\right]$ caused by the unknown part of the averaged dissipative secondorder self-forces. For $n=3$, the 3.5PN known spin-independent part of the flux at the next leading order in the mass ratio is treated as unknown.

reason we focus on large $\nu / M$ is that, as will be discussed in Append. A the dephasing from the known PN terms is already well suppressed below $1 \mathrm{rad}$ if $\nu / M$ is small enough. Thus, the PN convergence for small $\nu / M$ does not affect the following discussion.

Notice also that $x_{0}$ becomes smaller and smaller for larger $\nu / M$. The 2.5PN case is marginal, in the sense that the entire range of $x$ contributes almost equally. By contrast, the contribution near the ISCO dominates for the corrections at the $3 \mathrm{PN}$ order or higher. Although the PN convergence is not clearly seen for $q>0.9$, we should note that the phase error is never extremely enhanced for some particular post-Newtonian order, largely exceeding the values for the $3 \mathrm{PN}$ or 3.5PN order, upon which we mainly focus in the following discussion.

\section{B. The expected dephasing from the unkown higher-order post-Newtonian terms}

Now we move on to our main issue: evaluating the dephasing due to the yet-unknown higher -order PN terms, $\Delta \Phi\left[\mathcal{L}_{\text {full }}^{[0]}+\right.$ $\left.\tilde{\mathcal{L}}_{3.5 \mathrm{PN}}^{[1]}, \tilde{\mathcal{L}}_{>3.5 \mathrm{PN}}^{[1]}\right]$, based on the idea for the extrapolation proposed in Sec. [IV The scaling argument will tell us that the dephasings coming from the respective PN terms at the leading order in the mass ratio will be roughly proportional to the corresponding next-leading-order dephasings. Namely,

$$
\Delta \Phi\left[\mathcal{L}_{0 \mathrm{PN}}^{[0]}, \tilde{\mathcal{L}}_{n \mathrm{PN}}^{[0]}-\mathcal{L}_{0 \mathrm{PN}}^{[0]}\right]: \Delta \Phi\left[\tilde{\mathcal{L}}_{n \mathrm{PN}}^{[0]}, \tilde{\mathcal{L}}_{>n \mathrm{PN}}^{[0]}\right] \approx \Delta \Phi\left[\tilde{\mathcal{L}}_{\text {full }}^{[0]}, \tilde{\mathcal{L}}_{n \mathrm{PN}}^{[1]}\right]: \Delta \Phi\left[\mathcal{L}_{\text {full }}^{[0]}+\tilde{\mathcal{L}}_{n \mathrm{PN}}^{[1]}, \tilde{\mathcal{L}}_{>n \mathrm{PN}}^{[1]}\right]
$$

will hold. Recall that $\tilde{\mathcal{L}}_{n \mathrm{PN}}^{[0]}$ consists of the terms at the leading order in mass ratio up to the same $n \mathrm{PN}$ order that is available in $\tilde{\mathcal{L}}_{n \mathrm{PN}}^{[1]}$.

Here, we have three basic options in our choice of flux: the Taylor flux, the exponential resummed flux and the hybrid flux. (Recall that the hybrid flux is defined as such that is identical to the exponential resummed flux at the leading order in the mass ratio.) On one hand, the Taylor flux is problematic since it can be negative before reaching ISCO for some PN orders, say 2.5PN, with a modelate value of the spin parameter as we mentioned earlier. In such cases the phase before the plunge is not well defined. On the other hand, the difference between the exponential resummed flux and the hybrid flux is negligible. Therefore, we here consider the hybrid flux only.

Using Eq. 222, the residual dephasing estimated by

$$
\Delta \Phi_{\text {guess }}\left[\mathcal{L}_{\text {full }}^{[0]}+\tilde{\mathcal{L}}_{n \mathrm{PN}}^{[1]}, \tilde{\mathcal{L}}_{>n \mathrm{PN}}^{[1]}\right]:=\frac{\Delta \Phi\left[\tilde{\mathcal{L}}_{n \mathrm{PN}}^{[0]}, \tilde{\mathcal{L}}_{>n \mathrm{PN}}^{[0]}\right]}{\Delta \Phi\left[\mathcal{L}_{0 \mathrm{PN}}^{[0]}, \tilde{\mathcal{L}}_{n \mathrm{PN}}^{[0]}-\mathcal{L}_{0 \mathrm{PN}}^{[0]}\right]} \Delta \Phi\left[\tilde{\mathcal{L}}_{\text {full }}^{[0]}, \tilde{\mathcal{L}}_{n \mathrm{PN}}^{[1]}\right],
$$

with $n=3$ and $n=3.5$, is depicted in Fig. 4 The trend of the estimated dephasing is independent of the truncated PN order. The point of Fig. [4 is that the residual dephasing is rather suppressed over the whole range of the binary parameters. Indeed, the residual dephasing is at most about $10 \mathrm{rad}$ in the case of $3 \mathrm{PN}$ truncation. In the case of 3.5PN truncation, the maximum value of the residual dephasing is bigger by a factor of two or so. Since the plot is given in radians, the value must be divided by $2 \pi$ to translate it into the number of cycles. From the above results, one may say that the residual dephasing due to the yet-unknown 
PN corrections at the next leading order in the mass ratio $\Delta \Phi_{\text {guess }}\left[\mathcal{L}_{\text {full }}^{[0]}+\tilde{\mathcal{L}}_{n \mathrm{PN}}^{[1]}, \tilde{\mathcal{L}}_{>n \mathrm{PN}}^{[1]}\right]$ is not negligible, adopting one cycle as the criterion for the significant dephasing.

The curves in Fig.4 are, roughly speaking, broken power-law curves. The shallower slope on the right corresponds to the range in which the initial separation of the binary is sufficiently large. In this case the suppression due to the mass ratio in the higher-order correction is compensated for by the longer duration spent by the orbit in a given frequency band. By contrast, the steeper slope on the left corresponds to the region in which the initial separation is small. In this case, the total number of cycles is cut off by the observation period, and hence it does not increase for smaller $\nu / M$. As a result, the dephasing rapidly decreases for smaller $\nu / M$ below the critical value determined by the observation period.

In Fig. 4. we also see a tiny bump around $\nu M_{\odot} / M \approx 10^{-10}-10^{-11}$ for some values of $q$. The reason why this bump appears at this position can be understood from the observation that $\tilde{\mathcal{L}}_{n \mathrm{PN}}^{[0]}-\mathcal{L}_{0 \mathrm{PN}}^{[0]}$ crosses zero within the domain of the integral, $x_{0}<x<x_{\mathrm{ISCO}}$ when the bump appears. When $\tilde{\mathcal{L}}_{n \mathrm{PN}}^{[0]}-\mathcal{L}_{0 \mathrm{PN}}^{[0]}$ crosses zero, the factor in the denominator of Eq. (23) $\Delta \Phi\left[\mathcal{L}_{0 \mathrm{PN}}^{[0]}, \tilde{\mathcal{L}}_{n \mathrm{PN}}^{[0]}-\mathcal{L}_{0 \mathrm{PN}}^{[0]}\right]$ suffers from more or less accidental suppression even though we take the absolute value in the integrand of $\Delta \Phi$ in Eq. (20). We denote the value of $x$ at which the flux $\tilde{\mathcal{L}}_{n \mathrm{PN}}^{[0]}-\mathcal{L}_{0 \mathrm{PN}}^{[0]}$ crosses zero by $x_{\text {cross }}$. Then, if $x_{0} \ll x_{\text {cross }}$ or $x_{0} \gg x_{\text {cross }}$, this suppression does not produce much effect on the estimate of $\Delta \Phi\left[\mathcal{L}_{0 \mathrm{PN}}^{[0]}, \tilde{\mathcal{L}}_{n \mathrm{PN}}^{[0]}-\mathcal{L}_{0 \mathrm{PN}}^{[0]}\right]$. Therefore, the suppression becomes significant only for $x_{0} \approx x_{\text {cross }}$. The value of $x_{\text {cross }}$ is rather close to the value at ISCO, $x_{\mathrm{ISCO}}$, but typically not extremely close to it. Hence, $x_{0} \approx x_{\text {cross }}$ occurs when $x_{0}$ is neither extremely close to ISCO nor very small like $x_{0} \ll 1$, which corresponds to the break of the curves at $\nu M_{\odot} / M \approx 10^{-10}-10^{-11}$. Since now we find that this tiny bump is to be attributed to an accidental zero in $\tilde{\mathcal{L}}_{n \mathrm{PN}}^{[0]}-\mathcal{L}_{0 \mathrm{PN}}^{[0]}$, this bump would be regarded as an artificial feature. If it is fair to remove the bumps from Fig. 4, we will find that the significantly large dephasing (in the sense of exceeding one cycle) will be expected only for $\nu M_{\odot} / M \gtrsim 10^{-9}$.

\section{Unknown dephasing expected when we know the lower-PN-order nonlinear spin-dependent terms in the energy flux}

In the preceding subsection, we find that the dephasing caused by the unknown PN higher-order terms at the next leading order in mass ratio can be $O(10)$ rad or more. However, these unknown terms in the flux include nonlinear spin terms at the 3 $\mathrm{PN}$ or lower order and all spin-dependent terms at the 3.5PN order, and hence we have excluded the corresponding terms from $\tilde{\mathcal{L}}_{n \mathrm{PN}}^{[0]}$, which appears in the expression Eq. (23). In Fig. 4] relatively large dephasing is observed for large $|q|$, for which the nonlinear spin terms will be important.

Here we consider how the estimate presented in the preceding subsection is modified once we obtain all the spin-dependent terms up to $3 \mathrm{PN}$ or 3.5PN order. What we need to evaluate is the expression obtained by removing from (23), i.e.,

$$
\Delta \Phi_{\text {guess }}\left[\mathcal{L}_{\text {full }}^{[0]}+\mathcal{L}_{n \mathrm{PN}}^{[1]}, \mathcal{L}_{>n \mathrm{PN}}^{[1]}\right]:=\frac{\Delta \Phi\left[\mathcal{L}_{n \mathrm{PN}}^{[0]}, \mathcal{L}_{>n \mathrm{PN}}^{[0]}\right]}{\Delta \Phi\left[\mathcal{L}_{0 \mathrm{PN}}^{[0]}, \mathcal{L}_{n \mathrm{PN}}^{[0]}-\mathcal{L}_{0 \mathrm{PN}}^{[0]}\right]} \Delta \Phi\left[\mathcal{L}_{\text {full }}^{[0]}, \tilde{\mathcal{L}}_{n \mathrm{PN}}^{[1]}\right]
$$

Here, one may think that $\tilde{\mathcal{L}}_{n \mathrm{PN}}^{[1]}$ in the factor $\Delta \Phi\left[\mathcal{L}_{n \mathrm{PN}}^{[0]}, \tilde{\mathcal{L}}_{n \mathrm{PN}}^{[1]}\right]$ should have also been replaced with $\mathcal{L}_{n \mathrm{PN}}^{[1]}$. Since we do not have the expression for $\mathcal{L}_{n \mathrm{PN}}^{[1]}$ at hand, we cannot perform this replacement. However, the difference between $\mathcal{L}_{n \mathrm{PN}}^{[1]}$ and $\tilde{\mathcal{L}}_{n \mathrm{PN}}^{[1]}$ will not be significant, because their dominant PN terms are common.

The resultant dephasing $\Delta \Phi_{\text {guess }}\left[\mathcal{L}_{\text {full }}^{[0]}+\mathcal{L}_{n \mathrm{PN}}^{[1]}, \mathcal{L}_{>n \mathrm{PN}}^{[1]}\right]$ is depicted in Fig. 5. In contrast to the Fig. 4. except for the nearextremal-spin case $(q=0.998)$ with 3.5PN truncation $(n=3.5)$, the residual dephasing for large $\nu / M$ evaluated by using the hybrid flux does not exceed $1 \mathrm{rad}$. The residual dephasing exceeds $1 \mathrm{rad}$ only around the peak, which is likely to be an artifact due to the suppression in $\Delta \Phi\left[\mathcal{L}_{0 \mathrm{PN}}^{[0]}, \mathcal{L}_{n \mathrm{PN}}^{[0]}-\mathcal{L}_{0 \mathrm{PN}}^{[0]}\right]$ similarly to the previous case. The above result indicates that the dominant source of the error caused by the unknown part of the flux is in the spin-dependent terms at the lower PN orders up to the $3 \mathrm{PN}$ or 3.5PN order. Namely, the knowledge of nonlinear spin-dependent terms in the energy flux is crucial for reducing the uncontrolled dephasing caused by the averaged dissipative second-order self-forces. In addition, once we obtain these spin dependent terms of the energy flux, the exponential resummation will improve its accuracy to a level almost sufficient for the detection of $\mathrm{E}(\mathrm{I}) \mathrm{MRIs}$ in the whole interesting parameter region.

\section{SUMMARY AND CONCLUSION}

To evaluate the second-order self-forces in the context of the black hole perturbation is a challenging issue motivated by the goal to extract information from extreme (intermediate) mass-ratio inspirals [E(I)MRIs]. In this paper we have assessed the dephasing of the GW waveform from quasicircular E(I)MRIs, caused by the averaged dissipative part of the second-order self-forces, focusing on giving an order-of-magnitude estimate of the influence of the yet-unknown higher-order post-Newtonian 

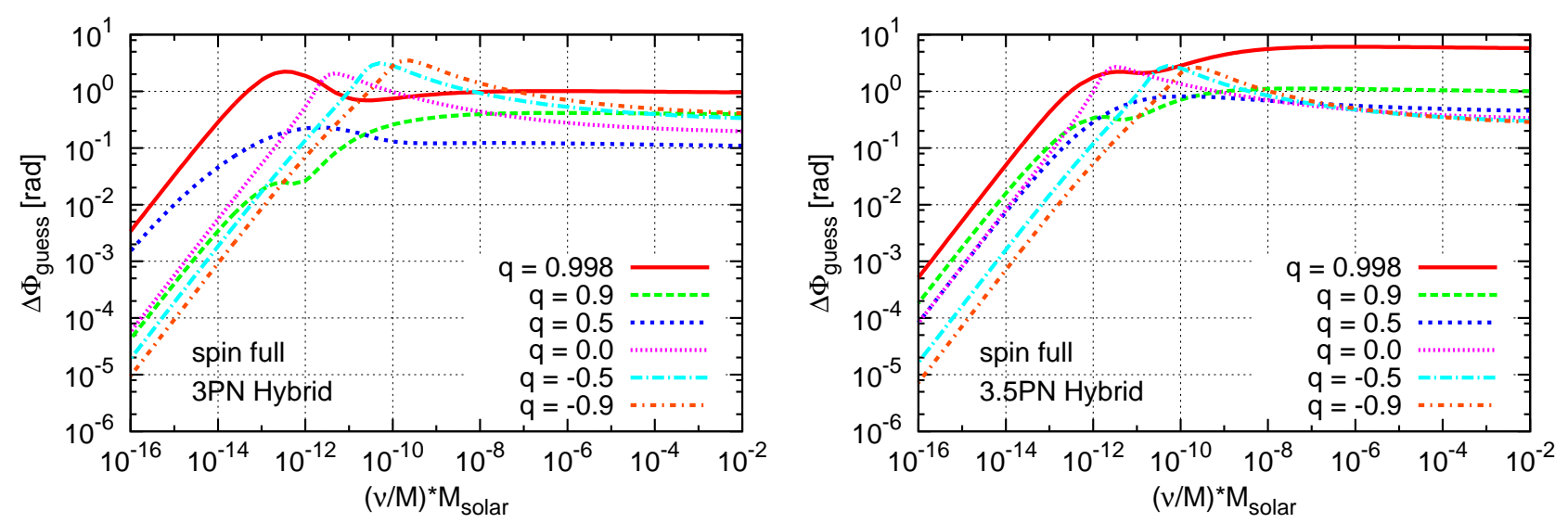

FIG. 5: The expected residual dephasing $\Delta \Phi_{\text {guess }}\left[\mathcal{L}_{\text {full }}^{[0]}+\mathcal{L}_{n \mathrm{PN}}^{[1]}, \mathcal{L}_{>n \mathrm{PN}}^{[1]}\right]$ when we assume that the spin-dependent terms at the lower PN orders are all known.

(PN) corrections. Using the balance argument, the dephasing is related to the correction to the the emitted energy flux. Although it will still require further efforts to calculate the second-order self-forces using the black hole perturbation, they are partially already known from the standard PN calculation. We gave an estimate of how much dephasing will be caused by the yet-unknown higher-order PN terms for the last $1 \mathrm{yr}$ of inspiral before the plunge, exhaustively exploring the whole possible parameter region of E(I)MRIs.

To give a guess for the yet-unknown higher-order PN terms, we first introduced a simple resummation method for the energy flux, which we call the exponential resummation. This is simply obtained by exponentiating the energy flux and truncating it at the known PN order in the exponent. This resummation has three merits: it ensures the positivity of the energy flux, it accelerates the PN convergence, and it can be applicable if we just know the PN Taylor flux. Since the PN Taylor flux in the test particle limit with large $q$ is known to be negative outside the innermost stable circular orbit (ISCO) at several PN orders, the total phase before the plunge becomes ill defined. Hence, we cannot discuss the amplitude of the higher PN corrections by using the PN Taylor flux. The exponential resummation solves this problem and improves the PN convergence in terms of the total phase before the plunge. When we discuss the finite mass corrections to the energy flux, we can combine the idea of the exponential resummed flux with the known exact energy flux in the test particle limit. We also proposed such a phenomenological energy flux, which we call the hybrid flux.

To examine the dephasing from the unknown part of the averaged dissipative second-order self-forces, we need some extrapolation. Using the brand-new 8 PN energy flux in the test particle limit[? ] that includes all spin-dependent terms, we discovered that the order of the magnitude of the absolute values of the coefficients up to the $8 \mathrm{PN}$ order approximately follows a simple scaling law, which is what we expect when the actual convergence radius of the PN expansion is at the light ring radius, irrespective of the value of dimensionless spin parameter $q$. Since there is no reason to expect that the energy flux diverges outside the light-ring radius even if we take into account the finite mass corrections for quasicircular E(I)MRIs, we are motivated to assume that the ratio between the magnitude of the terms at $O\left(\nu^{0}\right)$ and $O(\nu)$ at the same PN order will roughly stay independent of the PN order.

Based on this assumption, we estimated the unknown portion of the energy flux that comes from the higher PN terms at the next leading order in the mass ratio and evaluated the residual dephasing due to them. We find that the residual dephasing may exceed one cycle for $\nu M_{\odot} / M \gtrsim 10^{-10}$ and spin of the Kerr blackhole $|q|>0.5$, assuming a $1 \mathrm{yr}$ observation period. For some parameters, we found a little enhancement of the estimated dephasing at $\nu M_{\odot} / M \approx 10^{-10}-10^{-11}$. Since this enhancement is likely to be attributed to a mild accidental cancellation in the factor in the denominator of the estimator of the residual dephasing that we adopted, it might be fair to remove the bump that arises for this reason. Then, the residual dephasing exceeds one cycle only for relatively large $O(\nu / M)$ with $|q|>0.5$. Even for rather extreme case like $q=0.998$ (the Thorne limit), the expected residual dephasing is at most a few cycles or so.

In the unknown flux, nonlinear spin terms at lower PN orders are also included since they are not yet calculated in the context of PN approximation even at the leading order in the PN expansion. As large residual dephasing is expected only for large $|q|$, one may suspect that the residual dephasing might be dramatically reduced once we know all the nonlinear spin-dependent terms up to the $3 \mathrm{PN}$ or $3.5 \mathrm{PN}$ order. Therefore we made analogous plots for the expected residual dephasing assuming that the nonlinear spin-dependent terms are also available. We found that the expected residual dephasing is further suppressed to be less than $1 \mathrm{rad}$ for $|q|<0.9$, as we expected. Even in the extreme case with $q=0.998$, the expected dephasing becomes less than 1 rad if we use $3 \mathrm{PN}$ truncation to evaluate the residual. We think the reason why 3.5PN truncation is worse is simply because, for large $q$ close to unity, ISCO is very close to the light-ring radius, and hence the PN convergence is very poor. Thus, the resulting 
estimate of the residual also fluctuates by a large order of magnitude in this limit. However, even if we rely on 3.5PN truncation, the expected dephasing is at most about one cycle for $q=0.998$.

Although a 1 yr observation period is assumed in all the above estimates, the results do not depend much on it. A change in the observation time varies the relation between $x_{0}$ and $\nu / M$, and hence the curves in Figs. 4 and 5 are just shifted horizontally leftward. The expected dephasing becomes large only for large $\nu / M$, but $x_{0}$ is already sufficiently small in this case. Therefore the effect of changing the observation period for large $\nu / M$ appears only in Fig. 4. in which lower-order PN terms are contributing. In fact, one can see that the curves in Figs. 5 are already saturated for large $\nu / M$.

To conclude, the residual dephasing caused by the unknown averaged dissipative part of the second-order self-forces is estimated to be at most a few cycles. What is more, this dephasing is mostly to be attributed to the lower-PN-order nonlinear spin-dependent terms. If the PN expansion to the $3 \mathrm{PN}$ or 3.5PN order is completed including the dependence on the black hole spin, the residual is expected to be further reduced. We think that this conclusion will not change even if we are slightly underestimating the dephasing.

It should be stressed that we are not trying to claim that all the second-order terms can be neglected for detection. The known part of the averaged dissipative second-order self-forces might be necessary even just for detection. Moreover, when the parameter extraction from EMRIs is concerned, even a small dephasing in principle gives a bias. Hence, to what extent the higher-order corrections are needed crucially depends on the accuracies required by the physics that we wish to extract and also on the signal-to-noise ratio.

As a final remark, we should note that there is a possibility that we might be underestimating the residual dephasing by an order of magnitude, since the PN convergence is not very smooth, especially for positive large $q$. Even if our estimate turns out to be a good approximation of the real magnitude, we still have a chance to have a golden event with a large signal-to-noise ratio. In that case, one cycle or even one radian may not be a sufficient accuracy for the template waveforms. Then, the dissipative part of the second-order self-forces that cannot be captured by the PN expansion becomes necessary to extract the best physics from the observational data. Also we should mention that we focused on quasicircular E(I)MRIs in this paper, but our scaling argument will not apply anymore for significantly eccentric orbits, since there are many modes whose frequency exceeds the naive PN convergence radius that will be given by the absolute value of the first complex quasi-normal mode frequency. Therefore, the hybrid energy flux cannot be expected to remain a good approximation near the plunge for such systems. Hence, our analysis does not at all discourage the study on the averaged dissipative part of the second-order self-forces. Our claim is that it will be possible to perform nearly the best analyses of most (nearly) quasicircular E(I)MRIs without waiting for the full development of our knowledge about the averaged dissipative part of the second-order self-forces.

\section{Acknowledgments}

S.I. acknowledges the support of the Grant-in-Aid for JSPS Fellows, No. 24-4281. R.F. is grateful for the support of the European Union FEDER funds, the Spanish Ministry of Economy and Competitiveness (Project No. FPA2010-16495 and No. CSD2007-00042) and the Conselleria d'Economia Hisenda i Innovacio of the Govern de les Illes Balears. R.F. also appreciates the warm hospitality at the Yukawa Institute for Theoretical Physics where part of this work was completed. H.T. is supported by the Grand-in-Aid for Scientific Research (No 23540309), and T.T. is supported by the Grand-in-Aid for Scientific Research (No. 21111006, No. 21244033, No.24103001, and No. 24103006). Finally, this work was supported by the Grant-in-Aid for the Global COE Program "The Next Generation of Physics, Spun from Universality and Emergenc" from the Ministry of Education, Culture, Sports, Science and Technology of Japan.

\section{Appendix A: Dephasing from the known post-Newtonian terms}

This appendix is dedicated to finding the dephasing from the available PN terms in the next leading order in the mass ratio. In the bulk of our paper, we introduced three different types of PN flux formulas: Taylor, exponential resummed and hybrid, but there is no qualitative difference among $\Delta \Phi_{\mathrm{PN}}\left[\mathcal{L}_{\text {full }}^{[0]}, \tilde{\mathcal{L}}_{n \mathrm{PN}}^{\mathrm{T}[1]}\right], \Delta \Phi_{\mathrm{PN}}\left[\mathcal{L}_{\text {full }}^{[0]}, \tilde{\mathcal{L}}_{n \mathrm{PN}}^{\exp [1]}\right]$ and $\Delta \Phi_{\mathrm{PN}}\left[\mathcal{L}_{\text {full }}^{[0]}, \tilde{\mathcal{L}}_{n \mathrm{PN}}^{\text {hyb }[1]}\right]$, as long as we choose the exact numerical flux $\mathcal{L}_{\text {full }}^{[0]}$ as the reference flux. This is because the significant difference among various fluxes appears only in the higher PN residual parts, such as those discussed in Sec. $\mathrm{V}$ Therefore, we show only $\Delta \Phi_{\mathrm{PN}}\left[\mathcal{L}_{\text {full }}^{[0]}, \tilde{\mathcal{L}}_{3 \mathrm{PN}}^{\text {hyb }[1]}\right]$ in Fig. 6. We stress that what we show is the dephasing caused by the terms not at the $3 \mathrm{PN}$ order but up to the 3PN order. The main message of Fig. 6is that the corrections to the phase of GWs due to the higher-order terms in the mass ratio are suppressed below $1 \mathrm{rad}$, even if we include the leading order of the PN expansion- for a binary with $\nu M_{\odot} / M<10^{-12}$, say, when the mass of the central Kerr black hole is greater than $3 \times 10^{6} M_{\odot}$ with the satellite mass fixed to $10 M_{\odot}$. This holds irrespective of the value of the dimensionless spin parameter $q$. This plot clearly denies the naive statement that the effects of higher PN corrections should be important for a more massive central black hole because the satellite stays near the ISCO for a longer period. 


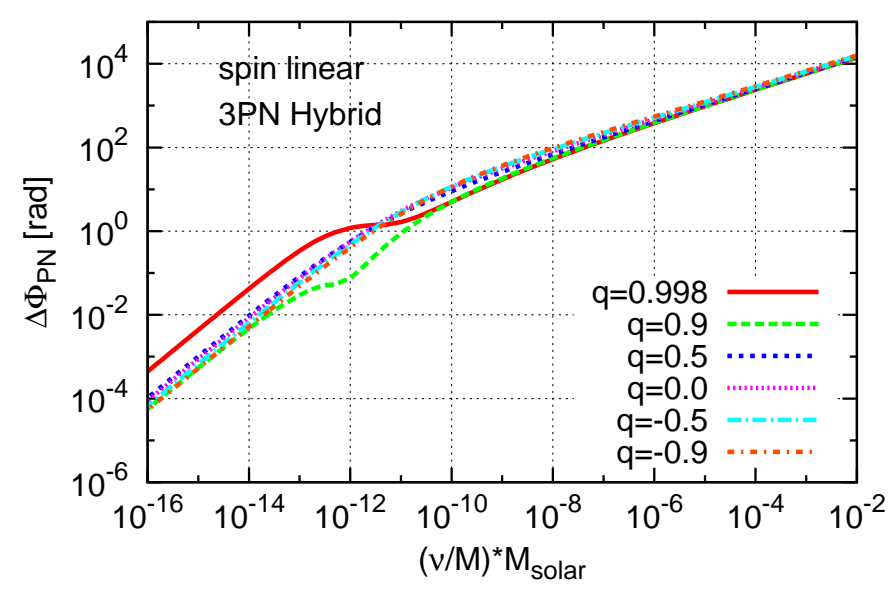

FIG. 6: The dephasing caused by the known terms up to the $3 \mathrm{PN}$ order at the next leading order in the mass ratio, $\Delta \Phi_{\mathrm{PN}}\left[\mathcal{L}_{\text {full }}^{[0]}, \tilde{\mathcal{L}}_{3 \mathrm{PN}}^{\mathrm{hyb}[1]}\right]$, for various dimensionless spin parameters $q$ of a Kerr black hole, based on the 3 PN hybrid flux 16.

As for results in the literature for comparison, for a binary with $M_{\mathrm{BH}}:=10^{6} M_{\odot}, q=0$ and $\nu=10^{-5}$. Heurte and Gair[38] reported $1.5 \mathrm{rad}$ dephasing after the last $1 \mathrm{yr}$ of inspiral based on the $2 \mathrm{PN}$ Taylor flux, while Yunes et al. [39] reported $0.71 \mathrm{rad}$ dephasing after the last 11.5 months of inspiral for the same binary parameters. Following our definition of the dephasing, we calculate $\Delta \Phi_{\mathrm{PN}}\left[\mathcal{L}_{\text {full }}^{[0]}, \tilde{\mathcal{L}}_{2 \mathrm{PN}}^{\mathrm{hyb}[1]}\right]$ to find $1.46 \mathrm{rad}$, which we think is in good agreement with the previous results, within the variance due to the different definition of dephasing.

\section{Appendix B: Dephasing due to the horizon absorption flux}

In the bulk of our manuscript, we neglected the flux absorbed by the horizon. In the test particle limit, Tagoshi et al. [69] and Yunes et al. [39] have already shown that the energy flux absorbed through the horizon cannot be neglected, leading to a large dephasing, especially when the Kerr black hole has large spin. However, this does not mean that the corrections due to the horizon absorption flux at the next leading order in the mass ratio are also important. Naively, the standard PN formalism is not suitable for calculating the horizon absorption flux since the black hole horizon is beyond the reach of the standard PN expansion, although there is a direction to evaluate the horizon absorption flux by relating it with the tidal field around each black hole[70, 71].

In this appendix, we briefly address the dephasing caused by the flux absorbed through the horizon in the test particle limit to see what can be said about the dephasing due to the horizon absorption flux at the next leading order in the mass ratio. The flux absorbed through the horizon in the test particle limit is expanded as [69]

$$
\mathcal{L}_{n \mathrm{PN}}^{[0, \mathrm{H}]}(x):=\frac{32}{5} \nu^{2} x^{5}\left\{\left(-\frac{1}{4} q-\frac{3}{4} q^{3}\right) x^{5 / 2}+\left(-q-\frac{33}{16} q^{3}\right) x^{7 / 2}+O\left(x^{4}\right)\right\},
$$

and the exact numerical flux is also calculable [54]. We denote the contribution from the first term in the curly brackets in the above expression by $\mathcal{L}_{2.5 \mathrm{PN}}^{[0, \mathrm{H}]}$ and the remaining part of the horizon flux by $\mathcal{L}_{>2.5 \mathrm{PN}}^{[0, \mathrm{H}]}$, in a similar manner as before. As we mentioned above, the horizon absorption flux at the next leading order in the mass ratio is still under development, but the part that requires the second-order metric perturbation should start with $1 \mathrm{PN}$ order higher than the leading terms in the test particle limit, starting at the $3.5 \mathrm{PN}$ order. Hence, we adopt $\mathcal{L}_{>2.5 \mathrm{PN}}^{[0, \mathrm{H}]}$, excluding $\mathcal{L}_{2.5 \mathrm{PN}}^{[0, \mathrm{H}}$, as the counterpart of the unknown horizon absorption flux at the next leading order in the mass ratio. Under this consideration, we compare the dephasing due to the horizon absorption flux

$$
\Delta \Phi\left[\mathcal{L}_{\text {full }}^{[0]}+\mathcal{L}_{2.5 \mathrm{PN}}^{[0, \mathrm{H}]}, \mathcal{L}_{>2.5 \mathrm{PN}}^{[0, \mathrm{H}]}\right]
$$

to that coming from the PN truncation $\Delta \Phi\left[\mathcal{L}_{n \mathrm{PN}}^{\exp [0]}, \mathcal{L}_{>n \mathrm{PN}}^{\exp [0]}\right]$ for $n=3$ or $n=3.5$ in the test particle limit. The results are plotted in FIG.7for $q= \pm 0.9$. 

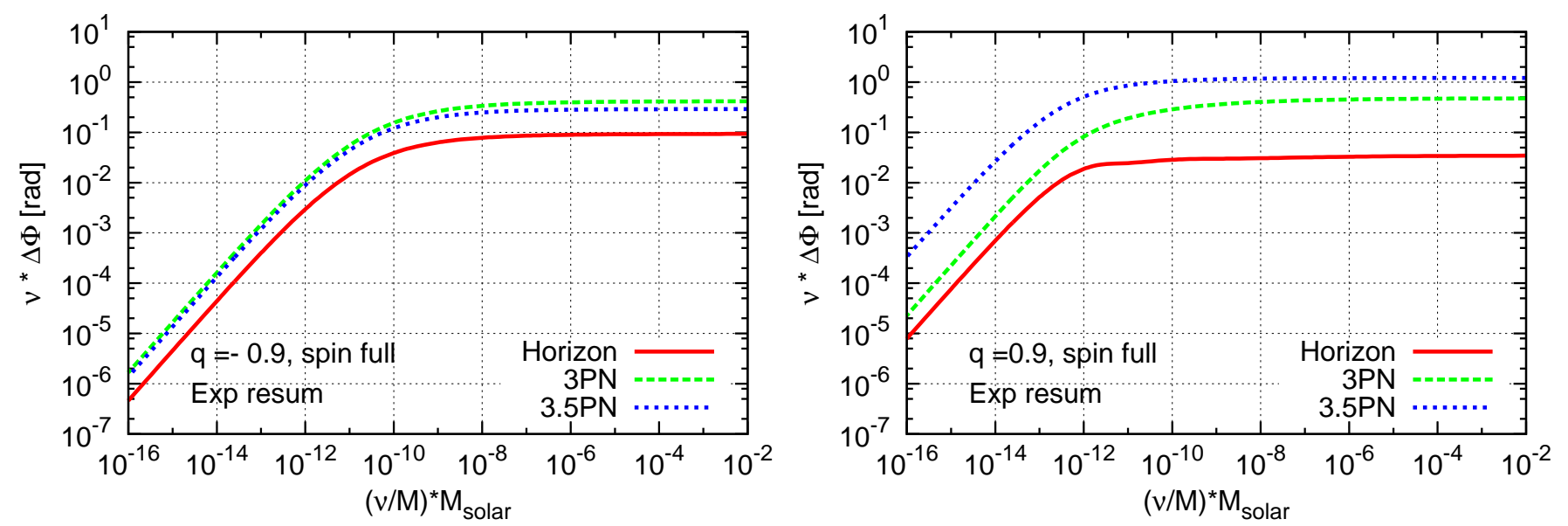

FIG. 7: The dephasing $\nu \Delta \Phi\left[\mathcal{L}_{\text {full }}^{[0]}+\mathcal{L}_{2.5 \mathrm{PN}}^{[0, \mathrm{H}]}, \mathcal{L}_{>2.5 \mathrm{PN}}^{[0, \mathrm{H}]}\right]$ due to the horizon absorption flux and the dephasing $\nu \Delta \Phi\left[\mathcal{L}_{n \mathrm{PN}}^{\exp [0]}, \mathcal{L}_{>n \mathrm{PN}}^{\exp [0]}\right] \operatorname{due}$ to the higher PN corrections, for $n=3$ and $n=3.5$, and $q= \pm 0.9$.

Fig. 7 indicates that $\nu \Delta \Phi\left[\mathcal{L}_{\text {full }}^{[0]}+\mathcal{L}_{2.5 \mathrm{PN}}^{[0, \mathrm{H}]}, \mathcal{L}_{>2.5 \mathrm{PN}}^{[0, \mathrm{H}]}\right]$ stays, at most, $O\left(10^{-1}\right)$ rad for the entire $\mathrm{E}(\mathrm{I}) \mathrm{MRI}$ parameter region. Indeed, it is, at most, about $1 / 10$ of $\nu \Delta \Phi\left[\mathcal{L}_{n \mathrm{PN}}^{\exp [0]}, \mathcal{L}_{>n \mathrm{PN}}^{\exp [0]}\right]$. For large $q$, the smallness of the effect of the horizon flux relative to the higher-order PN corrections can be understood from the work by Hughes[72], who numerically found that the energy flux absorbed by a Kerr black hole is , at best, ten times smaller than that emitted to infinity, with the aid of the fact that the PN expansion shows a poor convergence near the ISCO for large $q$. Thus, we expect that the residual dephasing due to the horizon absorption flux will be minor compared with the higher PN flux to infinity, and also in the next leading order in the mass ratio.

[1] Y. Mino, M. Sasaki and T. Tanaka, Phys. Rev. D 55, 3457 (1997) |gr-qc/9606018].

[2] T. C. Quinn and R. M. Wald, Phys. Rev. D 56, 3381 (1997) |gr-qc/9610053].

[3] C. R. Galley, B. L. Hu and S. -Y. Lin, Phys. Rev. D 74, 024017 (2006) [gr-qc/0603099].

[4] C. R. Galley and B. L. Hu, Phys. Rev. D 79, 064002 (2009) [arXiv:0801.0900 [gr-qc]].

[5] S. E. Gralla and R. M. Wald, Class. Quant. Grav. 25, 205009 (2008) [Erratum-ibid. 28, 159501 (2011)] [arXiv:0806.3293 [gr-qc]].

[6] A. Pound, Phys. Rev. D 81, 024023 (2010) [arXiv:0907.5197[gr-qc]].

[7] E. Rosenthal, Phys. Rev. D 72, 121503 (2005) |gr-qc/0508050|; 73, 044034 (2006) |gr-qc/0602066]; 74, 084018 (2006) [gr-qc/0609069|.

[8] S. Detweiler, Phys. Rev. D 85, 044048 (2012) [arXiv:1107.2098 [gr-qc]].

[9] A. Pound, Phys. Rev. Lett. 109, 051101 (2012) [arXiv:1201.5089 [gr-qc]].

[10] S. E. Gralla, Phys. Rev. D 85, 124011 (2012) [arXiv:1203.3189][gr-qc]].

[11] A. Pound, Phys. Rev. D 86, 084019 (2012) |arXiv:1206.6538 [gr-qc]].

[12] L. Barack, Class. Quant. Grav. 26, 213001 (2009) arXiv:0908.1664 [gr-qc]].

[13] E. Poisson, A. Pound and I. Vega, Living Rev. Rel. 14, 7 (2011) arXiv:1102.0529 [gr-qc]].

[14] T. Hinderer and E. E. Flanagan, Phys. Rev. D 78, 064028 (2008) [arXiv:0805.3337][gr-qc]].

[15] T. Tanaka, Prog. Theor. Phys. Suppl. 163, 120 (2006) [gr-qc/0508114|.

[16] Y. Mino, Prog. Theor. Phys. 113, 733 (2005) |gr-qc/0506003|.

[17] E. E. Flanagan and T. Hinderer, Phys. Rev. Lett. 109, 071102 (2012) [arXiv:1009.4923 [gr-qc]].

[18] J. Gair, N. Yunes and C. M. Bender, J. Math. Phys. 53, 032503 (2012) [arXiv:1111.3605] [gr-qc]].

[19] E. E. Flanagan, S. A. Hughes and U. Ruangsri, arXiv:1208.3906 [gr-qc].

[20] D. V. Gal'tsov, J. Phys. A 15, 3737 (1982).

[21] Y. Mino, Phys. Rev. D 67, 084027 (2003) gr-qc/0302075].

[22] N. Sago, T. Tanaka, W. Hikida, and H. Nakano, Prog. Theor. Phys. 114, 509 (2005) |gr-qc/0506092].

[23] N. Sago, T. Tanaka, W. Hikida, K. Ganz, and H. Nakano, Prog. Theor. Phys. 115, 873 (2006) |gr-qc/0511151].

[24] S. Drasco and S. A. Hughes, Phys. Rev. D 73, 024027 (2006) |gr-qc/0509101].

[25] L. M. Burko, Phys. Rev. D 67, 084001 (2003) |gr-qc/0208034]; Class. Quant. Grav. 22, S847 (2005).

[26] A. Pound, E. Poisson and B. G. Nickel, Phys. Rev. D 72, 124001 (2005) [gr-qc/0509122].

[27] A. Pound and E. Poisson, Phys. Rev. D 77, 044013 (2008) @arXiv:0708.3033 [gr-qc]].

[28] J. R. Gair, Class. Quant. Grav. 26, 094034 (2009) [arXiv:0811.0188 [gr-qc]].

[29] J. Thornburg, arXiv:1006.3788 [gr-qc]; arXiv:1102.2857 [gr-qc]. 
[30] L. Barack and A. Ori, Phys. Rev. D 61, 061502 (2000) [gr-qc/9912010].

[31] L. Barack, Y. Mino, H. Nakano, A. Ori, and M. Sasaki, Phys. Rev. Lett. 88, 091101 (2002) [gr-qc/0111001].

[32] Y. Mino, H. Nakano, and M. Sasaki, Prog. Theor. Phys. 108, 1039 (2003) |gr-qc/0111074].

[33] S. L. Detweiler and B. F. Whiting, Phys. Rev. D 67, 024025 (2003) |gr-qc/0202086].

[34] L. Barack and A. Ori, Phys. Rev. D 67, 024029 (2003) |gr-qc/0209072|.

[35] N. Warburton, S. Akcay, L. Barack, J. R. Gair and N. Sago, Phys. Rev. D 85, 061501 (2012) [arXiv:1111.6908][gr-qc]].

[36] K. A. Lackeos and L. M. Burko, Phys. Rev. D 86, 084055 (2012) \arXiv:1206.1452][gr-qc]].

[37] A. G. Shah, J. L. Friedman and T. S. Keidl, Phys. Rev. D 86, 084059 (2012) arXiv:1207.5595 [gr-qc]].

[38] E. A. Huerta and J. R. Gair, Phys. Rev. D 79, 084021 (2009) [Erratum-ibid. D 84, 049903 (2011)] [arXiv:0812.4208 [gr-qc]].

[39] N. Yunes, A. Buonanno, S. A. Hughes, Y. Pan, E. Barausse, M. C. Miller, and W. Throwe, Phys. Rev. D 83, 044044 (2011) [arXiv:1009.6013 [gr-qc]].

[40] Z. Zhang, N. Yunes and E. Berti, Phys. Rev. D 84, 024029 (2011) arXiv:1103.6041 [gr-qc]].

[41] H. Tagoshi, M. Shibata, T. Tanaka, and M. Sasaki, Phys. Rev. D 54, 1439 (1996) [gr-qc/9603028].

[42] A. Le Tiec, A. H. Mroue, L. Barack, A. Buonanno, H. P. Pfeiffer, N. Sago, and A. Taracchini, Phys. Rev. Lett. 107, 141101 (2011) [arXiv:1106.3278 [gr-qc]].

[43] L. Blanchet, B. R. Iyer, and B. Joguet, Phys. Rev. D 65, 064005 (2002) [Erratum-ibid. D 71, 129903 (2005)] |gr-qc/0105098].

[44] L. Blanchet, G. Faye, B. R. Iyer, and B. Joguet, Phys. Rev. D 65, 061501 (2002) [Erratum-ibid. D 71, 129902 (2005)] [gr-qc/0105099].

[45] L. Blanchet, A. Buonanno and G. Faye, Phys. Rev. D 84, 064041 (2011) arXiv:1104.5659 [gr-qc]].

[46] R. Fujita, Prog. Theor. Phys. 127, 583 (2012) \arXiv:1104.5615 [gr-qc]]; Prog. Theor. Phys. 128, 971 (2012) [arXiv:1211.5535 [gr-qc]].

[47] H. Nakano, R. Fujita and N. Sago (private communication.)

[48] R. H. Boyer and R. W. Lindquist, J. Math. Phys. 8, 265 (1967).

[49] E. A. Huerta and J. R. Gair, Phys. Rev. D 84, 064023 (2011) [arXiv:1105.3567] [gr-qc]].

[50] E. A. Huerta, J. R. Gair and D. A. Brown, Phys. Rev. D 85, 064023 (2012) [arXiv:1111.3243 [gr-qc]].

[51] J. M. Bardeen, W. H. Press, and S. A. Teukolsky, Astrophys. J. 178, 347 (1972).

[52] R. P. Geroch and J. Winicour, J. Math. Phys. 22, 803 (1981).

[53] S. A. Teukolsky, Astrophys. J. 185, 635 (1973); W. H. Press and S. A. Teukolsky, Astrophys. J. 185, 649 (1973); S. A. Teukolsky and W. H. Press, Astrophys. J. 193, 443 (1974).

[54] R. Fujita and H. Tagoshi, Prog. Theor. Phys. 112, 415 (2004) |gr-qc/0410018; R. Fujita, W. Hikida and H. Tagoshi, Prog. Theor. Phys. 121, 843 (2009) [arXiv:0904.3810 [gr-qc]].

[55] R. Fujita and H. Tagoshi, Prog. Theor. Phys. 113, 1165 (2005) @arXiv:0904.3818 [gr-qc]].

[56] T. Damour, B. R. Iyer and B. S. Sathyaprakash, Phys. Rev. D 57, 885 (1998) [gr-qc/9708034].

[57] E. K. Porter and B. S. Sathyaprakash, Phys. Rev. D 71, 024017 (2005) |gr-qc/0406038].

[58] E. K. Porter, Phys. Rev. D 76, 104002 (2007) [arXiv:0706.0114 [gr-qc]].

[59] J. Carre and E. K. Porter, arXiv:1206.2509 [gr-qc].

[60] T. Damour, B. R. Iyer, and A. Nagar, Phys. Rev. D 79, 064004 (2009) [arXiv:0811.2069 [gr-qc]].

[61] R. Fujita and B. R. Iyer, Phys. Rev. D 82, 044051 (2010) [arXiv:1005.2266 [gr-qc]].

[62] Y. Pan, A. Buonanno, R. Fujita, E. Racine, and H. Tagoshi, Phys. Rev. D 83, 064003 (2011) arXiv:1006.0431 [gr-qc]].

[63] C. Cutler, E. Poisson, G. J. Sussman, and L. S. Finn, Phys. Rev. D 47, 1511 (1993).

[64] M. Sasaki and H. Tagoshi, Living Rev. Rel. 6, 6 (2003) [gr-qc/0306120|.

[65] H. Onozawa, Phys. Rev. D 55, 3593 (1997) [gr-qc/9610048].

[66] K. S. Thorne, Astrophys. J. 191, 507 (1974).

[67] E. Poisson, gr-qc/0410127

[68] J. L. Friedman, K. Uryu, and M. Shibata, Phys. Rev. D 65, 064035 (2002) [Erratum-ibid. D 70, 129904 (2004)] [gr-qc/0108070].

[69] H. Tagoshi, S. Mano, and E. Takasugi, Prog. Theor. Phys. 98, 829 (1997) |gr-qc/9711072|.

[70] K. Alvi, Phys. Rev. D 64, 104020 (2001) |gr-qc/0107080|.

[71] E. Poisson, Phys. Rev. D 70, 084044 (2004) |gr-qc/0407050].

[72] S. A. Hughes, Phys. Rev. D 61, 084004 (2000) [Erratum-ibid. D 63, 049902 (2001)] [Erratum-ibid. D 65, 069902 (2002)] [Erratum-ibid. D 67, 089901 (2003)] [gr-qc/9910091]. 